\title{
Symbol-level and Multicast Precoding for Multiuser Multiantenna Downlink: A
}

\section{State-of-the-art, Classification and Challenges}

\author{
Maha Alodeh, Member, IEEE, Danilo Spano , Student Member, IEEE, \\ Ashkan Kalantari Member, IEEE, Christos Tsinos Member, IEEE, \\ Dimitrios Christopoulos Member, IEEE, Symeon Chatzinotas
}

Senior Member, IEEE, Björn Ottersten, Fellow Member, IEEE

\begin{abstract}
Precoding has been conventionally considered as an effective means of mitigating or exploiting the interference in the multiantenna downlink channel, where multiple users are simultaneously served with independent information over the same channel resources. The early works in this area were focused on transmitting an individual information stream to each user by constructing weighted linear combinations of symbol blocks (codewords). However, more recent works have moved beyond this traditional view by: i) transmitting distinct data streams to groups of users and ii) applying precoding on a symbol-persymbol basis. In this context, the current survey presents a unified view and classification of precoding techniques with respect to two main axes: i) the switching rate of the precoding weights, leading to the classes of block-level and symbol-level precoding, ii) the number of users that each stream is addressed to, hence unicast, multicast, and broadcast precoding. Furthermore, the classified techniques are compared through representative numerical results to demonstrate their relative performance and
\end{abstract}

Maha Alodeh, Danilo Spano, Ashkan Kalantari, Christos Tsinos, Symeon Chatzinotas and Björn Ottersten are with Interdisciplinary Centre for Security Reliability and Trust (SnT) at the University of Luxembourg, Luxembourg, e-mails:\{ maha.alodeh, danilo.spano, ashkan.kalantari, christos.tsinos, symeon.chatzinotas, and bjorn.ottersten@uni.lu\}, Dimitrios Christopoulos is with Newtec Satcom, Belgium, e-mail:\{dchr@newtec.eu\}.

This work is supported by Fond National de la Recherche Luxembourg (FNR) projects: SATellite SEnsor NeTworks for Spectrum Monitoring (SATSENT), Spectrum Management and Interference Mitigation in Cognitive Hybrid Satellite Network (SEMIGOD), Spectrum Management and Interference Mitigation in Cognitive Hybrid Satellite Network (INWIPNET), Energy and Complexity Efficient Millimeter-wave Large-Array Communications (ECLECTIC), Broadband/Broadcast Convergence through Intelligent Caching in 5G Satellite Networks (no. 10079323), and H2020 Project Shared Access terrestrial-satellite backhaul Network enabled by Smart Antennas (SANSA). 
uncover fundamental insights. Finally, a list of open theoretical problems and practical challenges are presented to inspire further research in this area. ${ }^{1}$

\section{Index Terms}

Directional modulation, multiuser MISO, symbol-level precoding, block-level precoding, channel state information, broadcast, unicast, multicast.

\section{INTRODUCTION}

Precoding has been a very prolific research area in recent years due to the promise of breaking the throughput gridlock of many interference-limited systems. The precoding performance gains originate from the combination of aggressive frequency reuse and suitable interference management techniques. Early works have focused in single-cell scenarios where the main limitation is intra-cell interference [1]-[6], while later works have also considered multi-cell and heterogeneous networks where inter-system interference [7]-[10] had to be considered as well. It should be noted that precoding has found applications in many practical communication systems, such as terrestrial cellular [7]-[11], satellite [12]-[14], Digital Subscriber Line (DSL) [15], powerline [16], [17], and visible light communications [18]-[20]. However, in order to provide a unifying view, this paper does not consider the peculiarities of each application area (e.g. channel, network architecture) but it rather focuses on a general communication model which can encompass the majority of precoding techniques.

Focusing on interference, this is one of the crucial and limiting factors in wireless networks. The concept of exploiting the users' spatial separation has been a fertile research domain for more than two decades [1], [6]. This can be implemented by adding multiple antennas at one or both communication sides. Multiantenna transceivers empower communication systems with more degrees of freedom that can boost the performance if the multiuser interference is mitigated properly. In this context, the term precoding can be broadly defined as the design of the transmitted signal to efficiently deliver the desired data stream at each user exploiting the multiantenna spatial degrees of freedom, data and channel state information while limiting the inter-stream interference.

In this survey, we use two major axes of classification depending on:

\footnotetext{
${ }^{1}$ The concepts of precoding and beamforming are used interchangeably throughout the paper.
} 
- The switching rate: how often the precoding coefficients are updated,

- The group size: the number of targeted users per information stream.

In the first classification, we differentiate between block-level and symbol-level precoding. In the former, the precoding coefficients are applied across block of symbols (or codewords), whereas in the latter they are applied on a symbol basis, i.e. switching with the baud rate. The second classification axis differentiates according to the requested service, namely among broadcast, unicast, and multicast. The first service type is known as broadcast, in which a transmitter has a common message to be sent to multiple receivers. In physical layer research, this service has been studied under the term of physical layer multicasting (i.e. PHY multicasting) [21][28]. Since a single data stream is sent to all receivers, there is no multiuser interference. However, precoding can still be used to improve the quality of service (QoS) across all users. The second service type is known as unicast, in which a transmitter has an individual message for each receiver. Due to the nature of the wireless medium and the use of multiple antennas, multiple simultaneous unicast transmissions are possible. In these cases, multiple streams are simultaneously sent, which motivates precoding techniques that mitigate the multiuser interference. From an information theoretic point of view, this service type has been studied using the broadcast channel [29]. Finally, the multicast service refers to the case where multiple messages are transmitted simultaneously but each message is addressed to a group of users. This case is also known as multigroup multicast precoding [30]-[42] ${ }^{2}$. It should be noted that broadcasting and unicasting present two extreme scenarios of multicasting. In unicasting, the number of users per group equals to 1 and the number of groups equals at maximum the number of antennas. In contrary to unicasting, the broadcasting aims at serve several users, however all of them belong to the same group. To capture this, we used the term multicast to highlight the generality of this services. The classification methodology is further detailed in Section I-B.

The paper is organized as the follows. This paper starts with introducing the scope of this survey by describing the communications model and the classification methodology in Section I. Then, it proceeds to the preliminaries in Section II. Section III describes in detail the fundamentals of block-level multicast precoding. Section IV describes the analog (directional modulation) and digital symbol-level precoding schems. Comparative studies between symbol-level and block

\footnotetext{
${ }^{2}$ It should be noted that alternative transmission strategies, such as rate-splitting and channels with both individual and common data will not be covered in this survey.
} 
level precoding as well as between block-level unicast, broadcast and multicast are conducted in Section V. Some challenges and open problems are thoroughly discussed VI. Finally, Section VII concludes the survey.

Notation: We use boldface upper and lower case letters for matrices and column vectors, respectively. $(\cdot)^{H},(\cdot)^{*}$ and $(\cdot)^{T}$ stand for the Hermitian transpose, conjugate and transpose of (.) respectively. $\mathbb{E}(\cdot)$ and $\|\cdot\|$ denote the statistical expectation and the Euclidean norm. $\angle(\cdot)$, $|\cdot|$ are the angle and magnitude of $(\cdot)$ respectively. $\mathcal{R}(\cdot), \mathcal{I}(\cdot)$ are the real and the imaginary part of $(\cdot)$. Finally, $\operatorname{tr}(\cdot)$ denotes the trace $(\cdot)$ and $[\cdot]_{m, n}$ denotes the element in the row $m$ and column $n$ of $[\cdot]$.

\section{A. Communication Model}

Let us assume that a base station (BS) is equipped with $N$ transmit antennas and wishes to transmit $Q$ number of symbol streams to $K$ single-antenna users (see Fig. 2-3). Adopting a baseband discrete memoryless model, the received signal at the $k$ th user for the symbol slot $t$ can be written as:

$$
y_{k}[t]=\mathbf{h}_{k}^{T} \mathbf{x}[t]+z_{k}[t]
$$

where $\mathbf{h}_{k}$ is an $N \times 1$ complex vector representing the channel of the $k$ th user, $\mathbf{x}[t]$ is an $N \times 1$ complex vector representing the output signal from the $N$ transmit antennas and $z_{k}[t]$ is a complex scalar representing the Additive White Gaussian Noise (AWGN) at receiver $k$ at instant $t$.

The above communication model can be equivalently written in a vector form as:

$$
\mathbf{y}[t]=\mathbf{H x}[t]+\mathbf{z}[t]
$$

where $\mathbf{y}[t]$ is a $K \times 1$ complex vector representing the received signal at all $K$ users at time $t$, $\mathbf{H}=\left[\mathbf{h}_{1} \ldots \mathbf{h}_{K}\right]^{T}$ is an $N_{t} \times K$ complex matrix representing the system channel matrix and $\mathbf{z}[t]$ is a $K \times 1$ complex vector representing the AWGN for all $K$ users at instant $t$.

It should be noted that in the context of this paper, we assume that each symbol stream is divided into blocks of $T$ symbols, while the channel matrix $\mathbf{H}$ remains constant for each block of symbols. In this context, $\mathbf{S}=\left[\mathbf{s}_{1} \ldots \mathbf{s}_{Q}\right]^{T}$ is an $Q \times T$ complex matrix aggregating the $T \times 1$ input symbol vectors $\mathbf{s}_{k}$ for each user or group $k$, which are assumed uncorrelated in time and space and having unit average power $\mathbb{E}_{t}\left[\mathbf{s}_{k}^{H}[t] \mathbf{s}_{k}[t]\right]=1$. Analogously, the $N_{t} \times T$ matrix $\mathbf{X}$ 
TABLE I: Summary of the System Model Parameters

\begin{tabular}{|l|l|}
\hline Parameter & Definition \\
\hline$N_{t}$ & Number of transmit antennas \\
\hline$K$ & Number of single antenna users \\
\hline$G$ & Number of groups \\
\hline$Q$ & $\begin{array}{l}\text { Number of symbol streams, for unicast } Q=K \\
\text { multicast } Q=G \text { and broadcast } Q=1\end{array}$ \\
\hline$T$ & The number of transmitted symbols in each block \\
\hline $\mathbf{h}_{k}$ & The channel between the BS and user $k$ \\
\hline $\mathbf{s}_{k}[t]$ & $\begin{array}{l}\text { The data stream (i.e. the set of symbols) dedicated to } \\
k \text { user or group/user at instant } t\end{array}$ \\
\hline $\mathbf{S}$ & $\begin{array}{l}\text { Complex matrix aggregating the data streams to be sent } \\
\text { to all users in the coherence time }\end{array}$ \\
\hline $\mathbf{x}[t]$ & The output vector from the antennas at $t$ \\
\hline $\mathbf{w}_{k}$ & The dedicated precoding vector to user $k$ or group $k$ \\
\hline$z_{k}[t]$ & The noise at receiver $k$ at instant $t$. \\
\hline$y_{k}[t]$ & The received signal at user $k$ at instant $t$. \\
\hline $\mathbf{y}[t]$ & $\begin{array}{l}\text { The concatenated received signals at all receiver at } \\
\text { instant } t .\end{array}$ \\
\hline$t$ & Time index \\
\hline
\end{tabular}

represents the block of output signals. In terms of system dimensions, we assume that $N \geq K$ and $K \geq Q$. In case $K>Q$, we assume that the users can be split in $Q$ equal groups of $G=K / Q$ users per group. The system parameters are summarized in Table III.

\section{B. Classification Methodology}

The adopted classification methodology is based switching rate and group size can be clarified as:

1) Block-vs Symbol-level precoding: The first classification axis is based on the switching rate of the precoding. Block-level precoding refers to techniques which apply precoding over symbol blocks. As a result, these techniques should use the side knowledge of the channel matrix $\mathbf{H}$, which includes estimates of the channel coefficients for all antenna-user pairs. In this case, precoding refers to designing the covariance matrix of the output signal vector $E_{t}\left[\mathbf{x}[t] \mathbf{x}^{H}[t]\right]$. The precoding changes with the channel and it is fixed over the symbol block. Symbol-level precoding refers to techniques where precoding is applied according to the baudrate. As a result, 


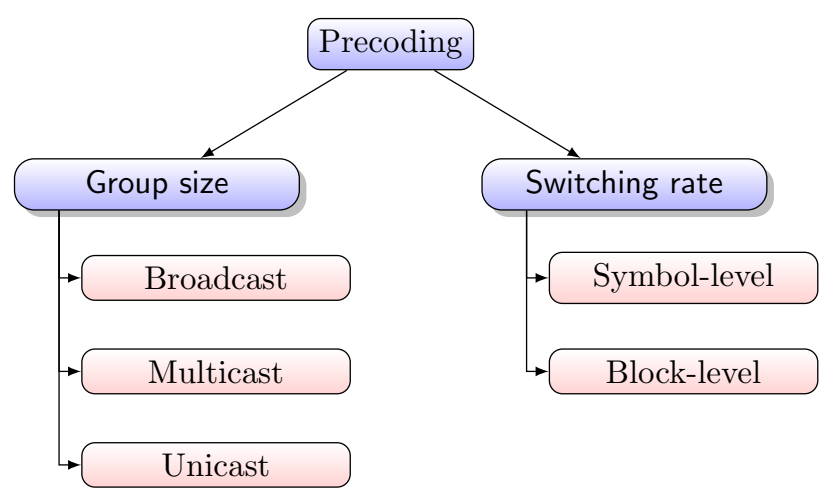

Fig. 1: Precoding Classification

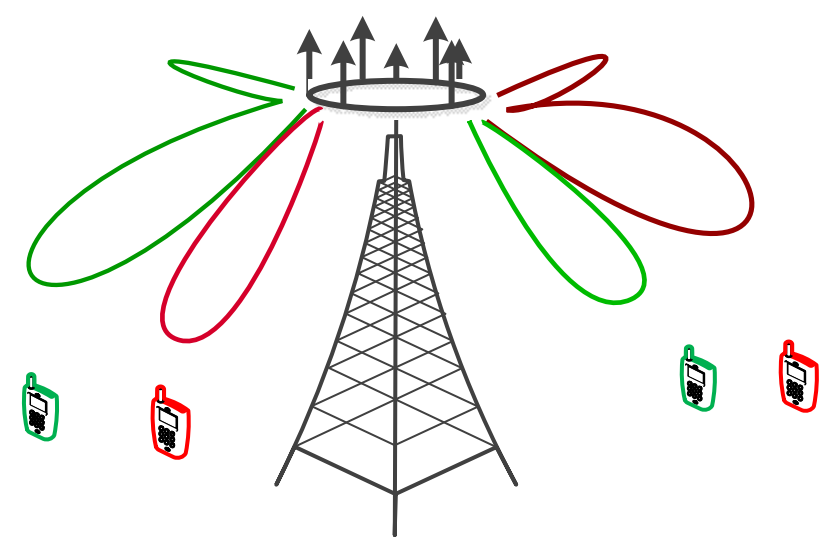

Fig. 2: System model for multicast precoding, the users with same color receive the same information.

the techniques can use the side knowledge of both the channel matrix $\mathbf{H}$ and the input symbol vector $\mathbf{s}_{k}[t]$. In this case, precoding refers to designing the actual output signal vector $\mathbf{x}[t]$.

2) Uni-/Multi-/Broad-cast Precoding: The second classification axis is based on the number of targeted users per symbol stream. Unicast precoding refers to cases where each symbol stream is destined to a single intended user, i.e. $M=K$. Broadcast precoding (a.k.a. PHYlayer Multicasting) refers to cases where a single symbol stream is destined to all users, i.e. $Q=1$. Multicast precoding refers to cases where each of $Q$ symbol streams is destined to $G$ groups of $K / Q$ intended users per group.

The precoder can be design to optimize performance metric whether the it is symbol-level or block-level. The two main metrics in the literature are transmitted Power and Quality of Service (SNR, rate etc). The usual approach is to optimize one metric while using the other as 


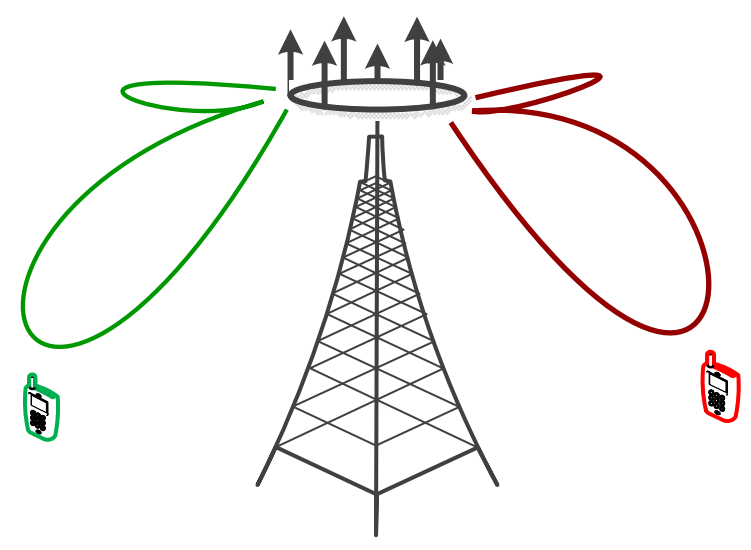

Fig. 3: System model for unicast precoding. Each user receives an individual message.

a constraint, e.g. power minimization under QoS constraints, QoS maximization under power constraints $^{3}$.

\section{Preliminaries}

\section{A. Power Metrics}

In this section, we formally define the basic power metrics that are going to be used in the remainder of this paper. By focusing on a specific symbol slot $t$ and a specific antenna $n$, the instantaneous per-antenna power is defined as $P_{n}=\left|x_{n}[t]\right|^{2}$. Similarly, by focusing on a specific symbol slot $t$ and all $N$ antennas, the instantaneous sum power is defined as $P=\|\mathbf{x}[t]\|^{2}=\operatorname{trace}\left(\mathbf{x}[t] \mathbf{x}^{H}[t]\right)$. By averaging across multiple symbol slots and considering all antennas, the average sum power is defined as $\bar{P}=E_{t}\left[\|\mathbf{x}[t]\|^{2}\right]=\operatorname{trace}\left(E_{t}\left[\mathbf{x}[t] \mathbf{x}^{H}[t]\right]\right)$. Finally by averaging across multiple symbol slots and considering a single antenna $n$, the average per-antenna power is defined as $\bar{P}_{n}=E_{t}\left[\left|x_{n}[t]\right|^{2}\right]=\left[E_{t}\left[\mathbf{x}[t] \mathbf{x}^{H}[t]\right]_{n n}\right.$.

One might wonder why we need so many different metrics. The answer is that each power metric serves a different purpose and can help address various implementation constraints or practical impairments. For example, average power provides an estimation of the long-term energy requirements, while instantaneous is a more detailed characterization, allowing to detect power spikes which could have unwanted side-effects. These side-effects include entering into the non-linear region of an amplifier or exceeding its maximum capability. Furthermore, the

\footnotetext{
${ }^{3}$ Often the two problems are dual and the power minimization is used as a stepping stone for solving the QoS maximization problem.
} 
per-antenna power metrics are meant to enable the investigation of each RF chain individually. More specifically, one could check how the power is distributed across the multiple antennas, since each RF chain usually has its own amplifier with individual impairments and limitations.

\section{B. QoS metrics (SNR, rate)}

In this section, we formally define the basic QoS metrics that are going to be used in the remainder of this paper. A basic QoS metric is the Signal to Interference and Noise Ratio (SINR), which enables us to characterize or optimize a ratio of desired to undesired power levels. However, an even more meaningful metric for communication systems is the rate. The dependence of rate to the SINR greatly depends on the employed input symbol distribution. The vast majority of approaches in the area of block-level precoding have used Gaussian inputs as a way of allowing the rate to scale logarithmically with the $\mathrm{SINR}^{4}$. However, in practical systems uniform discrete constellations (modulations) are commonly used in conjunction with adaptive modulation based on SINR thresholds to allow the rate scaling. This consideration complicates the rate calculation, because each symbol block might use a different modulation whose performance has to be studied separately. As we will see in section IV-B, the vast majority of symbol-level techniques have adopted the latter mode, since the detection regions of the discrete modulations can be more easily modeled.

\section{Block-level Unicast Precoding}

In this section, we briefly summarize some preliminaries on block-level unicast precoding, which is the most well-understood class in the literature. In this class, we could include dirty paper coding (DPC), which is an optimal non-linear technique based on known interference pre-cancellation which has been shown to achieve the MIMO downlink capacity [46], [47]. Tomlinson-Harashima precoding (THP), which is a suboptimal implementation of DPC [48], could also be considered in the class of block-level precoding. Nevertheless, hereafter the focus is on linear block-level precoding approaches, characterized by a lower complexity, thus being more suitable for practical implementations.

\footnotetext{
${ }^{4}$ It is worth mentioning some notable exceptions ([43], [44]-[45])
} 
In this framework, considering a block of $T$ symbol vectors to be conveyed to the users, modeled by the $Q \times T$ matrix $\mathbf{S}$, the corresponding block representing the output signals can be written as:

$$
\mathbf{X}=\mathbf{W S}
$$

The $N \times Q$ matrix $\mathbf{W}$ is the precoding matrix, applied to the entire information block $\mathbf{S}$. The precoding matrix can be written as $\mathbf{W}=\left[\mathbf{w}_{1} \ldots \mathbf{w}_{Q}\right]$, each column represents a precoding vector for the corresponding user.From this formalization, it is clear how the problem of block-level unicast precoding can be reduced to the problem of designing the precoding matrix $\mathbf{W}$, using the knowledge of the channel $\mathbf{H}$, in order to mitigate the interference. To this aim, the literature provides some closed-form as well as some solutions based on numerical optimization problems.

The most relevant closed-form solutions are zero-forcing (ZF) precoding [49], [50] and minimum mean square error (MMSE) precoding [2], [51]-[53]. ZF is one of the simplest suboptimal techniques, which decouples the multi-user channel into parallel single-user channel, thus canceling out the multi-user interference. To this aim, the $\mathrm{ZF}$ precoding matrix can be calculated as the pseudo-inverse of the channel matrix, as $\mathbf{W}=\mathbf{H}^{H}\left(\mathbf{H H}^{H}\right)^{-1}$. The ability of ZF precoding to cancel out the interference, makes it more appealing for the high SNR regime. However, since ZF does not take into account the effect of noise, it does not perform well in the low SNR regime (noise limited regime). MMSE precoding, on the other hand, takes into account both the interference and the noise in order to improve the system performance also in the noise limited scenarios [2]. The MMSE precoding matrix can be written as $\mathbf{W}=\mathbf{H}^{H}\left(\mathbf{H} \mathbf{H}^{H}+\alpha \mathbf{I}\right)^{-1}$, with $\alpha$ being a regularization parameter inversely proportional to the SNR. Because of its expression, the MMSE precoder is also referred to as regularized ZF (R-ZF) [2], [54], [55]. It is worth mentioning also maximum ratio transmission (MRT) precoding [56], aiming at maximizing the received SNR, which however is a suitable technique only in the noise limited regime, where the multi-user interference can be neglected.

The above mentioned closed-form solutions for precoding are effective and easy to implement. However, they do not allow to optimize the system with respect to specific objectives, or respecting specific constraints. In this regard, a number of optimization-based precoding techniques have been devised, so to enhance the flexibility of the transmitter. The literature on block-level precoding includes different optimization strategies for the precoding design. The optimal precoding strategy for the minimization of the transmitted average sum power, 
whilst guaranteeing some QoS targets at each user, was given in [57], [58]. For block level precoding, it can be shown that the average sum power is $\bar{P}=\sum_{j=1}^{M}\left\|\mathbf{w}_{j}\right\|^{2}$. Accordingly, the related optimization problem, which is optimally solved by semi-definite relaxation (SDR), can be written as follows:

$$
\begin{aligned}
\mathbf{W}(\mathbf{H}, \gamma)=\arg \min _{\mathbf{W}} & \sum_{j=1}^{M}\left\|\mathbf{w}_{j}\right\|^{2} \\
\text { s.t. } \quad & \frac{\left|\mathbf{h}_{j}^{T} \mathbf{w}_{j}\right|^{2}}{\sum_{k \neq j, k=1}^{M}\left|\mathbf{h}_{j}^{T} \mathbf{w}_{k}\right|^{2}+\sigma_{z}^{2}} \geq \gamma_{j} j \in K,
\end{aligned}
$$

where the inputs are the channel matrix and a vector $\gamma$ including the target SINR for the different users, and the output is the precoding matrix.

Another relevant precoding strategy aims at maximizing the minimum SINR across the users, under sum power constraints (SPC). This approach increases the fairness of the system, thus it is known as max-min fair optimization. The related optimization problem was solved in [59] based on the principles of uplink/downlink duality, and can be written as:

$$
\begin{array}{r}
\mathbf{W}(\mathbf{H}, P)=\arg \max _{\mathbf{W}} \min _{j} \frac{\left|\mathbf{h}_{j}^{T} \mathbf{w}_{j}\right|^{2}}{\sum_{k \neq j, k=1}^{M}\left|\mathbf{h}_{j}^{T} \mathbf{w}_{k}\right|^{2}+\sigma_{z}^{2}} \\
\text { s.t. } \quad \sum_{j=1}^{M}\left\|\mathbf{w}_{j}\right\|^{2} \leq P .
\end{array}
$$

Block-level precoding for unicast systems was extended in [60], [61] accounting for perantenna power constraints. In particular, it is worth mentioning that the average per-antenna power can be written as $\bar{P}_{n}=\left[\sum_{j=1}^{M} \mathbf{w}_{j} \mathbf{w}_{j}^{H}\right]_{n n}$. Moreover, further developments have been done considering per-antenna-array power constraints [62] and non-linear power constraints [63].

Unicast multiuser MIMO techniques have been proposed to utilize the spatial multiplexing gains of MIMO for different network capabilities such as multicell MIMO [64], cognitive radio [65], physical layer security [66], [67], simultaneous wireless information and power transfer [66], [68], etc.

\section{Block-Level Multicast Precoding}

A fundamental consideration of the multiuser unicast precoding is that independent data is addressed to each user. However, the new generation of multi-antenna communication standards has to adapt the physical layer design to the needs of the higher network layers. Examples of such cases include highly demanding applications (e.g. video broadcasting) that stretch the throughput 
limits of multiuser broadband systems. In this direction, physical layer (PHY) multicasting has the potential to efficiently address the nature of future traffic demand and has become part of the new generation of communication standards. PHY multicasting is also relevant for the application of beamforming without changing the framing structure of standards (cf. [33]).

\section{A. Multicast}

In the framework of block-level multicast precoding, we assume multiple interfering groups of users. In each group, each user receives a stream of common data. However, independent symbols are addressed to different groups and inter-group interferences comes into play. A unified framework for physical layer multicasting to multiple co-channel groups, where independent sets of common data are transmitted to groups of users by the multiple antennas, was given in [30][32]. Therein, the QoS and the fairness problems were formulated, proven NP-hard and solved for the sum power constrained multicast multigroup case. The QoS problem, aiming at minimizing the average sum transmit power, has been solved resorting to SDR, and can be written as:

$$
\begin{array}{ll}
\mathbf{W} & (\mathbf{H}, \gamma)=\underset{\mathbf{W}}{\operatorname{argmin}} \sum_{k=1}^{M}\left\|\mathbf{w}_{k}\right\|^{2} \\
\text { s.t. } & \frac{\left|\mathbf{h}_{i}^{T} \mathbf{w}_{k}\right|^{2}}{\sum_{l \neq k}\left|\mathbf{h}_{i}^{T} \mathbf{w}_{l}\right|^{2}+\sigma_{i}^{2}} \geq \gamma_{i}, \forall i \in \mathcal{G}_{k}, \forall k \in G,
\end{array}
$$

where $\mathbf{w}_{k} \in \mathbb{C}^{N_{t}}$, and $\mathcal{G}_{k}$ denotes the $k$-th group of users. The notation $\sum_{l \neq k}$ states that aggregate interference from all co-channel groups is calculated.

The weighted max-min fair problem under sum power constraints (SPC) has been solved via bisection over the QoS problem, and can be written as:

$$
\begin{aligned}
& \mathbf{W}(\mathbf{H}, P)=\quad \arg \min _{\alpha, \mathbf{W}} \alpha \\
& \text { s.t. } \quad \frac{1}{\gamma_{i}} \frac{\left|\mathbf{h}_{i}^{T} \mathbf{w}_{k}\right|^{2}}{\sum_{l \neq k}\left|\mathbf{h}_{i}^{T} \mathbf{w}_{l}\right|^{2}+\sigma_{i}^{2}} \geq \alpha, \\
& \forall i \in \mathcal{G}_{k}, k, l \in\{1 \ldots Q\} \text {, } \\
& \sum_{k=1}^{M}\left\|\mathbf{w}_{k}\right\|^{2} \leq P,
\end{aligned}
$$

where $\mathbf{w}_{k} \in \mathbb{C}^{N}$ and $t \in \mathbb{R}^{+}$. Different service levels between the users can be acknowledged in this weighted formulation. The problem receives as inputs the SPC $P$ and the target SINRs vector $\gamma=\left[\gamma_{1}, \gamma_{2}, \ldots \gamma_{K}\right]$. Its goal is to maximize the slack variable $t$ while keeping all SINRs 
above this value. Thus, it constitutes a max-min problem that guarantees fairness amongst users. Of particular interest is the case where the co-group users share the same target i.e. $\gamma_{i}=\gamma_{k}, \forall i \in$ $\mathcal{G}_{k}, k \in\{1 \ldots G\}$.

The weighted max-min fair problem has been addressed also accounting for per-antenna power constraints (PACs). In the related optimization problem, analogous to (7), the PACs read as $\left[\sum_{k=1}^{M} \mathbf{w}_{k} \mathbf{w}_{k}^{H}\right]_{n n} \leq P_{n}, \forall n \in\left\{1 \ldots N_{t}\right\}$. The weighted max-min fair problem with PACs has been solved through different approaches, as discussed hereafter.

1) SDR-based solution: The optimal multigroup multicast precoders when a maximum limit is imposed on the transmitted power of each antenna, have been derived in [34], [35]. Therein, a consolidated solution for the weighted max-min fair multigroup multicast beamforming problem under per-antenna constraints (PACs) is presented. This framework is based on SDR and Gaussian randomization to solve the QoS problem and bisection to derive an accurate approximation of the non-convex max min fair formulation. However, as detailed in [35], the PACs are bound to increase the complexity of the optimization problem and reduce the accuracy of the approximation, especially as the number of transmit antennas is increasing. These observations necessitate the investigation of lower complexity, accurate approximations that can be applied on large-scale antenna arrays, constrained by practical, per-antenna power limitations.

2) Successive Convex Approximation based solution: Inspired by the recent development of the feasible point pursuit (FPP) successive convex approximation (SCA) of non-convex quadratically constrained quadratic problems (QCQPs), as developed in [69], the work of [36] improved the max min fair solutions of [35] in terms of computational complexity and convergence. The FPP - SCA tool has been preferred over other existing approximations (for instance [69]) due to its guaranteed feasibility regardless of the initial state of the iterative optimization [69].

Apart from these two major approaches for solving multicast beamforming problems, an iterative technique recently appeared in literature [70]. In this paper, the QoS problem was cast in a equivalent form and then an iterative method based on alternating minimization was developed for its solution. This approach does not rely on optimization toolboxes and exhibits significant reduced computational complexity compared to the two other approaches while it achieves in general better performance than the SDR approach and very close to the SCA one. Furthermore, this approach was extended to the hybrid analog-digital transceivers' case which have growing interest the last years due to the recent developments in mmWave and Massive MIMO systems. Further approaches that investigate the potential of multicast beamforming schemes in hybrid 
transceivers or in general large array systems can be found in [36], [70]-[72]. Another SDR based approach has been proposed for multicast precoding in [38]. By properly shaping the beam patterns, intergroup interference can be avoided and the SINR at all receivers can be jointly controlled. DPC has been exploited to control the interference. Two problems have been solved in this context, the first problem is to minimize the transmit power under individual SINR constraints, the second problem is maximizing the minimum SINR under total power constraint.

Low complexity linear schemes have been proposed for the multigroup multicast case in [39]. They aim at providing tradeoff between computational complexity and performance. The proposed techniques are based on well-known algorithms, such as ZF, MMSE, SINR balancing tailored to the multicast case. The purpose is to overcome the high computational complexity of SDR based techniques without having significant loss in the overall performance.

The application of multicasting in multiuser MIMO environment has been proposed in different frameworks such as cognitive radio [73], simultaneous wireless information and power transfer [74], and hybrid analog digital beamforming for millimeter wave [75]. Recently, the capability of combining multicasting precoding with space time coding through multi-rank transmissions has been studied in [41], [73], [76]-[78], for more applications see Table. II.

\section{B. Broadcast}

Broadcast precoding can be seen as a special case of multicast, where we have a single group of users receiving the same data information. In this scenario, there is no interference since a single stream is sent to all users. In [21], the NP-hard broadcast precoding problem was accurately approximated by SDR and Gaussian randomization. The associated QoS problem can be written as:

$$
\begin{aligned}
\mathbf{w}(\mathbf{H}, \gamma)= & \arg \min _{\mathbf{w}}\|\mathbf{w}\|^{2} \\
& \text { s.t. } \frac{\left|\mathbf{h}_{j}^{T} \mathbf{w}\right|^{2}}{\sigma_{i}^{2}} \geq \gamma_{i}, \forall j \in K,
\end{aligned}
$$

where $\mathbf{w} \in \mathbb{C}^{N_{t}}$ represents the precoding vector for the unique transmitted data stream. Since no interference mitigation is included in the optimization problem (8), there is no dimensionality constraint, i.e. there is no constraints on the number of simultaneously served users, and the degrees of freedom of the problem are entirely leveraged to transmit information. An information theoretic capacity of broadcast is studied, along with the achievable rates using lower complexity 
transmission schemes in [22]. [22] focuses on the scaling of the capacity and achievable rates as the number of antennas and/or users is taken to infinity. Robustness for broadcast precoding is studied [23], where the goal is to design a beamformer that minimizes the transmit power while satisfying probabilistic signal-to-noise ratio (SNR) constraints for line of sight environment.

Low complexity algorithms have been proposed in [24], [25]. An approximate way for solving the problem based on a channel orthogonalization and local refinement has been proposed in [24]. An attractive performance-to-complexity tradeoff compared to the conventional multiple-antenna multicasting algorithms is illustrated in [24]. In [25], two scenarios have been tackled: a) the transmitter has perfect CSI for all users, and b) the transmitter has no initial CSI for any user. For the perfect CSI scenario, a new class of adaptive multicast beamforming algorithms is proposed comprising additive update, multiplicative update, and multiplicative update-successive linear approximation algorithms, with guaranteed convergence and state-of-the-art performance at low complexity. For scenarios where there is no initial channel state information at the transmitter, an online algorithm is developed that simultaneously learns the user channel correlation matrices and adapts the beamforming vector to maximize the minimum (long-term average) SNR among the users, using only periodic binary SNR feedback from each receiver. The online algorithm uses the analytic center cutting plane method to quickly learn the user correlation matrices with limited signaling overhead.

On the other hand, this problem has been studied from the viewpoint of minimizing outage probability under a transmit power constraint [26]. In this context, the channel can be modeled as a Gaussian mixture. The different Gaussian kernels model user clusters of different means (locations) and variances (spreads). It has been proven that minimizing outage probability subject to a transmit power constraint is an NP-hard problem when the number of Gaussian kernels (i.e. users), $K$, is greater than or equal to the number of transmit antennas, $N$. Moreover, it has also been shown that the problem is practically tractable for 2 - 3 Gaussian kernels through dimensionality reduction.

In the broadcast channel, the user having the worst SNR presents a bottleneck for the data transmission. In [28], some interesting insights into the bottleneck users, such as the maximum number of users to be served as well as the optimal beamformer when the transmitter has an arbitrary number of antenna elements. The max-min fair SNR problem is employed and the closed-form solutions are proposed from the weighted sum-SNR maximization perspective for different numbers of possible bottleneck users. 
TABLE II: Precoding Classification Based on the Group Size and the related Applications

\begin{tabular}{|c|c|c|c|}
\hline Precoding & Number of Groups & Number of Users & References \\
\hline Broadcast & 1 & $K$ & $\begin{array}{l}\text { Energy efficiency [21], [79], Fairness (Capacity)[21], [22], Robust[23], } \\
\text { [80], Physical-layer security [81], SWIPT[82], [83], Reduced complex- } \\
\text { ity [24], [25], [42], [84], Stochastic [85], finite alphabet [86], Constant } \\
\text { envelope [87], Physical-layer security [83], cognitive radio [88], [89], } \\
\text { Multirank [78], [85], [86], [90], Massive MIMO[91], Multicell [79] }\end{array}$ \\
\hline Unicast & $K$ & 1 & $\begin{array}{l}\text { Interference mitigation[6], [52], [61], [92]-[96], Interference exploita- } \\
\text { tion [94], [97]-[103], Energy efficiency[57]-[60], [104]-[106], sum } \\
\text { rate [107], Fairness [107], [108], Finite alphabets [43], [109], [110], } \\
\text { Robust [80], [111]-[121], Robust interference exploitation [122]- } \\
\text { [124], Constant envelope[125]-[127], Per-antenna optimization [60], } \\
\text { [61], [94], Physical layer security [66], [128], [129], cognitive radio } \\
\text { [65], [130], SWIPT [66], [68], Hybrid [131]-[135], Multirank[77], } \\
\text { Multibeam Satellite[13], [63], [136], Massive MIMO [137]-[140] }\end{array}$ \\
\hline Multicast & $G$ & $K / Q$ & $\begin{array}{l}\text { Fairness[30]-[33], [35], [36], [141], [142], [142]-[144], Energy effi- } \\
\text { ciency [145], Interference mitigation [39], Robust [40], [76], [146], } \\
\text { Stochastic [41], Coordinated [147], Per antenna [33]-[35], Relay } \\
\text { [148], SWIPT [74], Hybrid [75], Massive MIMO [36], [149], Satellite } \\
\text { [33], [37], Multirank[41], [73], coordinated multicell [106], [150], } \\
\text { [151] }\end{array}$ \\
\hline
\end{tabular}

The broadcast transmissions are studied in different contexts such as cognitive radio [80], simultaneous wireless and information power transfer [82], coordinated multicell network [147]. Moreover, multi-rank transmissions that combine space time coding and multicast beamforming are studied [41], [85], [86]. Physical-layer transmission techniques that combine multicast, unicast and broadcast have been proposed in [119], [152]-[158].

In 5G wireless network, we expect a dramatic increase in services and applications [159]. Employing an integrated framework of broadcast, multicast and unicast depending on the content of requested streams improves the efficiency of the wireless networks [154]-[158]. For example, using multicast solely, the rate of each link is limited by the worst user wasting a considerable link margin available for delivering extra information. To deal with this inefficiency, a multiuser MIMO system that enables a joint utilization of broadcast, unicast, and multicast is required. This can efficiently leverage the unused MIMO capability to send a broadcast stream or unicast streams concurrently with multicast ones, while ensuring no harm to the achievable rate of 
multicasting. Therefore, the throughput and energy efficiency of the whole network can be improved significantly. For more details about the classification based on the group size, please look at Table II.

\section{Symbol-LEVEl UnicAst PRECOding}

As observed in the block-level precoder class in Section III, precoding at the transmitter is used to mitigate the interference among the users' data streams. As another approach, the data and channel information can be used to perform symbol-level precoding at the transmitter. Symbollevel precoding guarantees interference-free communication at the expense of higher switching rate of the precoder. In the literature, symbol-level precoding paradigm has been proposed in two different research avenues, namely, directional modulation, via analog symbol-level precoding, developed in antenna and propagation domain and digital symbol-level precoding for constructive interference developed in signal processing and wireless communications. The solutions of both of these approaches are developed under the same context of channel and data dependent precoding, they originate from different areas and function under different system level models though. Thus, each one of them shares different advantages and disadvantages and comes with a different number of challenges that must be overcome towards the implementation of efficient transceiver solutions.

A transceiver based on the directional modulation concept consists of only a single RF chain which is fed by a local RF oscillator. The RF chain drives a network of phase shifters and variable gain amplifiers. In this technology, the antennas excitation weights change in the analog domain on a symbol basis, to create the desired phase and amplitude at the receiver sideinstead of generating the symbols at transmitter and sending them. While a single RF chain transceiver is highly desirable due to its simplistic structure and power consumption, there are several limitations regarding implementation difficulties and the lack of a strong algorithmic framework that need further study in the directional modulation field.

On the other hand, the digital symbol-level precoding for constructive interference uses digital precoding for signal design at the transmitter in order to create constructive interference at the receiver. The digital precoding happens before feeding the signal to the antenna array. Symbollevel precoding developed in the signal processing and wireless communications domain and the related techniques are more studied from an algorithmic point of view compared to the analog 


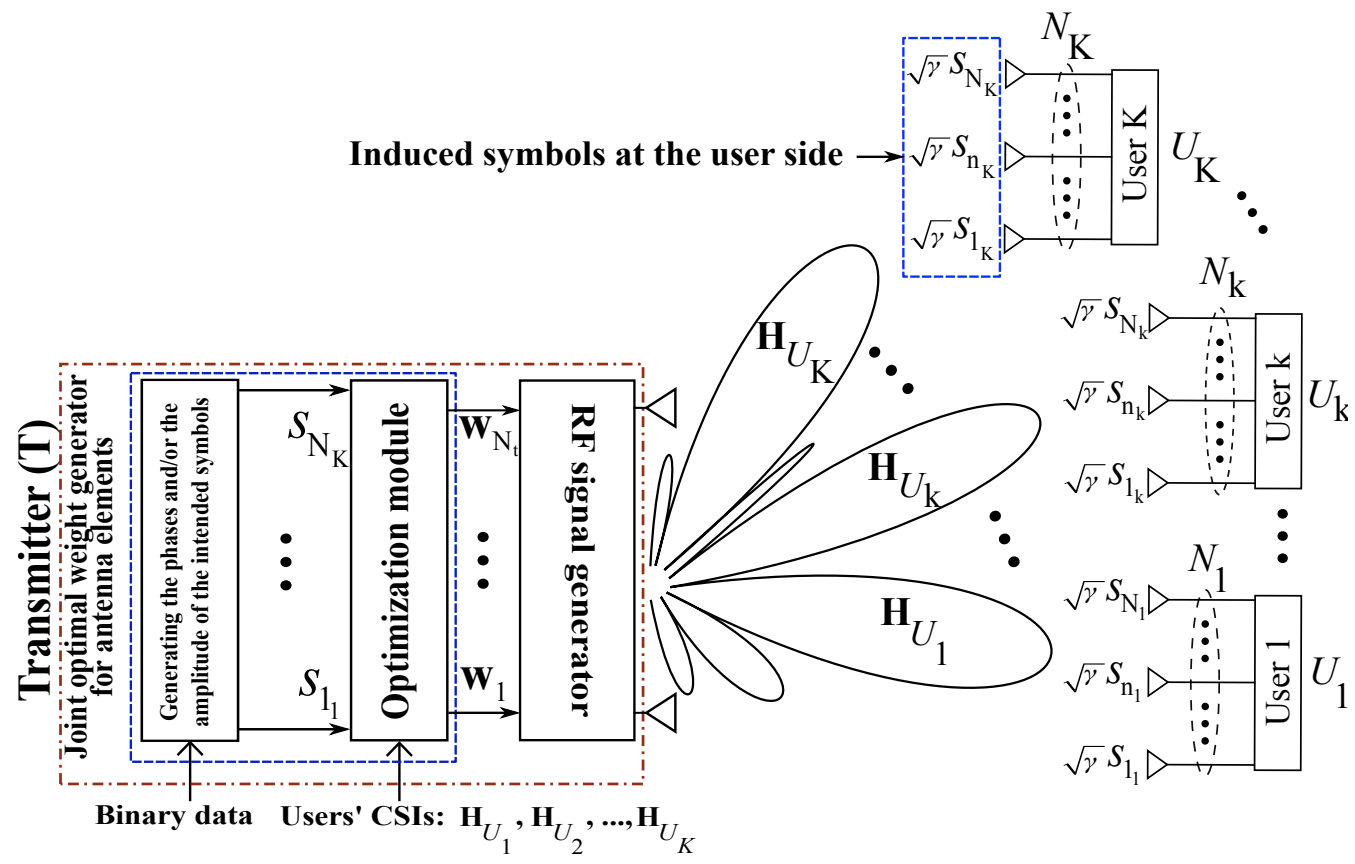

(a)

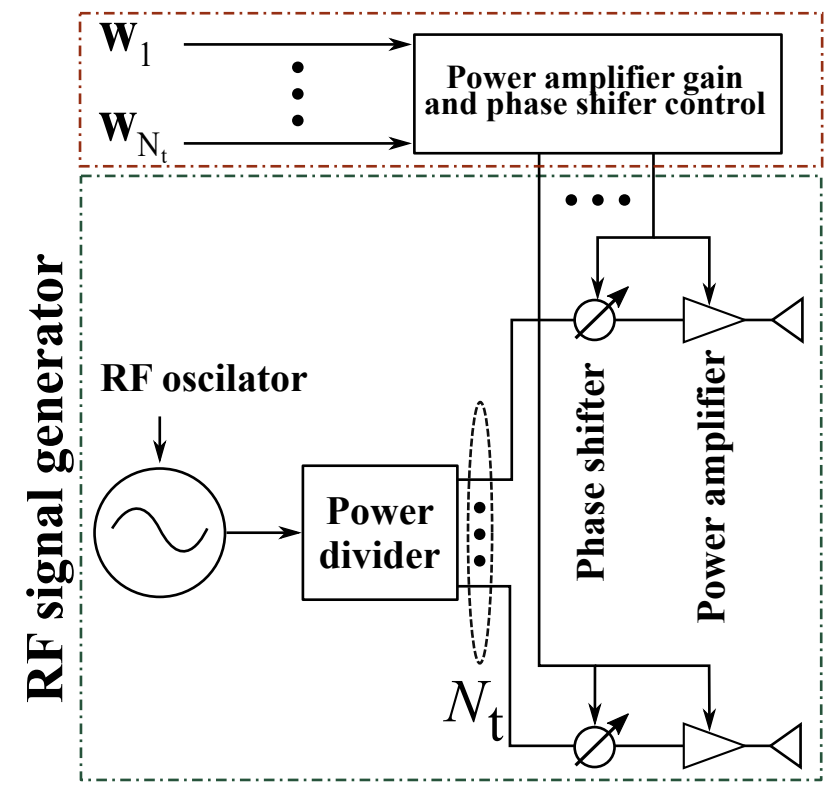

(b)

Fig. 4: 4(a): Generic structure of a directional modulation transmitter, and 4(b): Detailed schematic diagram for the RF signal generator section of a directional modulation transmitter (analog symbol-level precoding). 
symbol-level (directional modulation) based ones. On the contrary, they require a full digital transceiver, and thus there is difficulty in applying them in large antenna array systems.

In the following, a detailed description of directional modulation and digital symbol-level precoding are presented to show the differences and the similarities of the both schemes.

\section{A. Symbol-Level Precoding for Directional Modulation}

Directional modulation is an approach in which the users' channels and symbols are used to design the phase and amplitude of each antenna on a symbol basis such that multiple interferencefree or interference-limited symbols can be communicated with the receiver(s). After adjusting the array weights, the emitted radio frequency $(\mathrm{RF})$ signals from the array are modulated while passing through the fading channel. This is different from block-level precoding in which the transmitter generates the symbols and sends them after precoding [93], [160]. The benefit of directional modulation is that the precoder is designed such that the receivers' antennas can directly recover the symbols without CSI and equalization. The general structure of a MUMIMO directional modulation transmitter is shown in Fig. 4(a) where a MU-MIMO directional modulation transmitter communicates with $R$ multiple antenna users denoted by $U_{1}, \ldots, U_{k}, \ldots U_{K}$ with the corresponding number of antennas denoted by $N_{1}, \ldots, N_{K}$. The directional modulation transmitter communicates the symbols $S_{1_{i}}, \ldots, S_{n_{i}}, \ldots, S_{N_{i}}$ with the $i$-th user, denoted by $U_{i}$, for $i=1, \ldots, K$. Depending of the modulation type, the phase [161] or the phase and amplitude [162] of the intended symbols is generated and extracted using the binary data at the transmitter. The RF signal generator of the generic structure of Fig. 4(a) is detailed in Fig. 4(b) where a pair of phase shifter and power amplifier is used to tune the transmitted signal from each antenna.

Recently, there has been growing research interest on the directional modulation technology. Array switching approach at the symbol rate is used in [163]-[165] to induce the desired symbols at the receiver side. Specifically, the work of [163] uses an antenna array with a specific fixed delay in each RF chain to create the desired symbols by properly switching the antennas. The authors in [164] use an array where each element can switch to broadside pattern ${ }^{5}$, endfire pattern $^{6}$, or off status to create the desired symbols in a specific direction. The authors perform an extensive exhaustive search to find the best combination among the antenna patterns. In the

\footnotetext{
${ }^{5}$ Maximum radiation of an array directed normal to the axis of the array.

${ }^{6}$ Additional maxima radiation directed along the axis.
} 
work of [165], the elements of the array are switched to directionally modulate the binary phase shift keying (BPSK) constellation.

In another category, parasitic antenna is used to create the desired amplitude and phase in the far field by near field interactions between a driven antenna element and multiple reflectors [166][168]. As pioneers in this approach, [166], [167] use transistor switches or varactor diodes to change the reflector length or its capacitive load, respectively, when the channel is line of sight (LoS). This approach creates a specific symbol in the far field of the antenna towards the desired directions while randomizing the symbols in other directions due to the antenna pattern change. In connection with [166], [168] studies the far field area coverage of a parasitic antenna and shows that it is a convex region.

The authors of [169] suggest using a phased array at the transmitter, and employ the genetic algorithm to derive the phase values of a phased array in order to create symbols in a specific direction. The technique of [169] is implemented in [170] using a four element microstrip patch array where the genetic algorithm is used to derive the array phase in order to directionally modulated the symbols based quadratic phase shift keying (QPSK) modulation. The authors of [171] propose an iterative nonlinear optimization approach to design the array weights which minimizes the distance between the desired and the directly modulated symbols in a specific direction. In another paradigm, the authors of [172], divide the interference into static and dynamic parts and use genetic algorithms to design the array weights to directionally modulate the symbols.

In [173], baseband in-phase and quadrature-phase signals are separately used to excite two different antennas so that symbols are correctly transmitted only in a specific direction and scrambled in other directions. In another paradigm, [174] uses random and optimized codebook selection, where the optimized selection suppresses large antenna side lobes, in order to improve the security in a millimeter-wave large uniform linear antenna array system. The authors of [175] derive optimal array weights to get a specific bit error rate (BER) for QPSK modulation in the desired and undesired directions. The Fourier transform is used in [176], [177] to create the optimal constellation pattern for QPSK directional modulation. The work of [176] uses Fourier transform to create the optimal constellation pattern for QPSK directional modulation, while [177] uses Fourier transforms along with an iterative approach for QPSK directional modulation and constraining the far field radiation patterns. The effect of array structure on the directional modulation performance is investigated in [178]. The authors have shown that 
by increasing the space between the antennas of a two element array the symbol error rate can be improved for higher order $M$-ary phase shift key ( $M$-PSK) modulation such as 8-PSK. As an overview, [179] categorizes the directional modulation systems for QPSK modulation and discusses the proper metrics such as bit error rate for evaluating the performance of such systems. To overcome imperfect measurements, the authors of [180] propose a robust design for directional modulation in the presence of uncertainty in the estimated direction angle. The authors use minimum mean square error to minimize the distortion of the constellation points along the desired direction which improves the bit error rate performance.

In [177], [181]-[183] directional modulation is employed along with noise injection. The authors of [177], [181], [184] utilize an orthogonal vector approach to derive the array weights in order to directly modulate the data and inject the artificial noise in the direction of the eavesdropper. The work of [185] is extended to retroactive arrays ${ }^{7}$ in [182] for a multi-path environment. An algorithm including exhaustive search is used in [186] to adjust two-bit phase shifters for directly modulating information. The work of [184] introduces vector representations to link the vector paths and constellations. This helps figuring out the transmitter characteristics and the necessary and sufficient condition for directionally modulating symbols. It is shown that the directional modulation can be realized by adjusting the gain of the beamforming network.

The directional modulation concept is also extended to directionally modulate symbols to more than one destination. In [183], the singular value decomposition (SVD) is used to directionally modulate symbols in a two user system. The authors of [185] derive the array weights to create two orthogonal far field patterns to directionally modulate two symbols to two different locations and [187] uses least-norm to derive the array weights and directionally modulate symbols towards multiple destinations in a multi-user multi-input multi-output (MIMO) system. Later, [188] considers using ZF precoder to directionally modulate symbols and provide security for multiple single-antenna legitimate receivers in the presence of multiple single-antenna eavesdroppers. As a new approach, a synthesis free directional modulation system is proposed in [189] to securely communicate information without estimating the target direction.

The works of [161], [190] design the optimal symbol-level precoder for a security enhancing directional modulation transmitter in a MIMO fading channel to communicate with arbitrary

\footnotetext{
${ }^{7} \mathrm{~A}$ retroactive antenna can retransmit a reference signal back along the path which it was incident despite the presence of spatial and/or temporal variations in the propagation path.
} 
TABLE III: Precoding Classification Based on Switching Rate

\begin{tabular}{|l|l|}
\hline Precoding & References \\
\hline Block-level & $\begin{array}{l}\text { Interference mitigation [6], [30]-[32], [34], } \\
\text { [35], [38], [39], [39], [52], [57], [58], } \\
\text { [92], [95], [160], [191], [192] Energy ef- } \\
\text { ficiency [21], [30], [31], [57], [58], [60], } \\
\text { [104], Fairness [30], [32], [35], [35], [36], } \\
\text { [108], [193], Sum rate [33], [107], [109], } \\
\text { [110], [194], Robust [23], [40], [61], [61], } \\
\text { [76], [80], [111]-[114], [114], [116]-[118], } \\
\text { [129], [195], [196], Capacity[22], [29], } \\
\text { Physical-layer security [66], [67], [128], } \\
\text { [197], [198], SWIPT [66], [68], [74], [82], } \\
\text { [199], [200], Cognitive [65], [73], [80], } \\
\text { [80], [89], [130], [134], [201], [202], Co- } \\
\text { ordinated multicell [9], [64], [79], [106], } \\
\text { [145], [147], [151], [203], [204], Massive } \\
\text { MIMO [91], [155], [205], [206], Hybrid } \\
\text { analog/digital [70], [71], [75], [133]-[135], } \\
\text { [207], [208] } \\
\text { Energy efficiency [103], [209]-[215] Fair- } \\
\text { ness [210],[214], Sum rate [210], Ro- } \\
\text { bust [122]-[124], Interference exploitation } \\
\text { [94], [97]-[100], [100]-[103], [122]-[124], } \\
\text { [127], [209]-[214], [216], [217], Non- } \\
\text { linear channels [94], [216], SINR balanc- } \\
\text { ing [124], Constant envelope [87], [125]- } \\
\text { [127], [218], Physical-layer security [161], } \\
\text { [190], [219], [220], Simultaneous wireless } \\
\text { information and power transfer (SWIPT) } \\
\text { [221], cognitive [99], [102], [212] }\end{array}$ \\
\hline Symbol- \\
level
\end{tabular}

number of users and symbol streams. In addition, the authors derive the necessary condition for the existence of the precoder. The power and SNR minimization precoder design problems are simplified into a linearly-constrained quadratic programming problem. For faster design, an iterative approach as well as non-negative least squares formulation are proposed. 


\section{B. Symbol-level Precoding for Constructive Interference}

The interference among the multiuser spatial streams leads to a deviation of the received symbols outside of their detection region. Block-level precoding treats the interference as harmful factor that should be mitigated [49], [50], [57], [58], [60], [62], [160]. In this situation (see Fig. 5), the precoding cannot tackle the interference at each symbol and tries to mitigate the interference along the whole frame using only the knowledge of CSI, which manages to reduce the average amount of interference along the frame. For example, Fig. 5 represents the received signal at the first quadrant of QPSK modulation for block-level precoding. The target signal is the signal dedicated to the intended user and the interfering signal are the signals transmitted towards the other users. Since the precoding is applied once across the entire symbol block, each interfering signal cannot be treated individually. The resultant interference vector can push the target signal in any direction, therefore, the main optimization is to reduce the average power of interference to prevent it from pushing the target signals in the wrong direction.

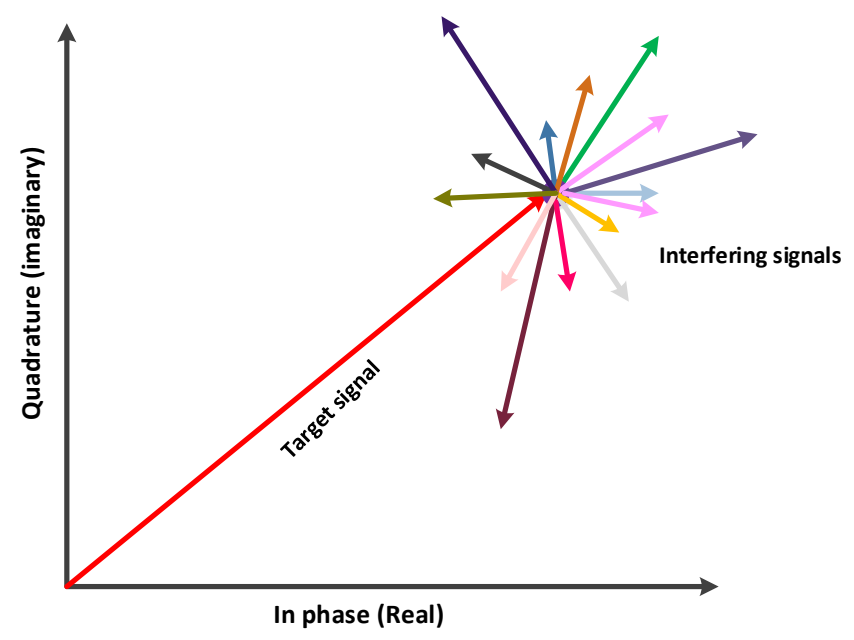

Fig. 5: Interference in Block-level Precoding. Interference can only be managed along whole frame.

During the past years several symbol-level processing techniques has been utilized in the multiuser MISO context [94], [97]-[103], [122]-[124], [127], [209]-[214], [216], [217]. A similar concept to the symbol-level precoding is the so-called constant envelop precoding that appeared recently in the literature [87], [125], [126], [222]-[228]. In these techniques, constant modulus constraints are set to the complex baseband signal of each transmit antenna which is 


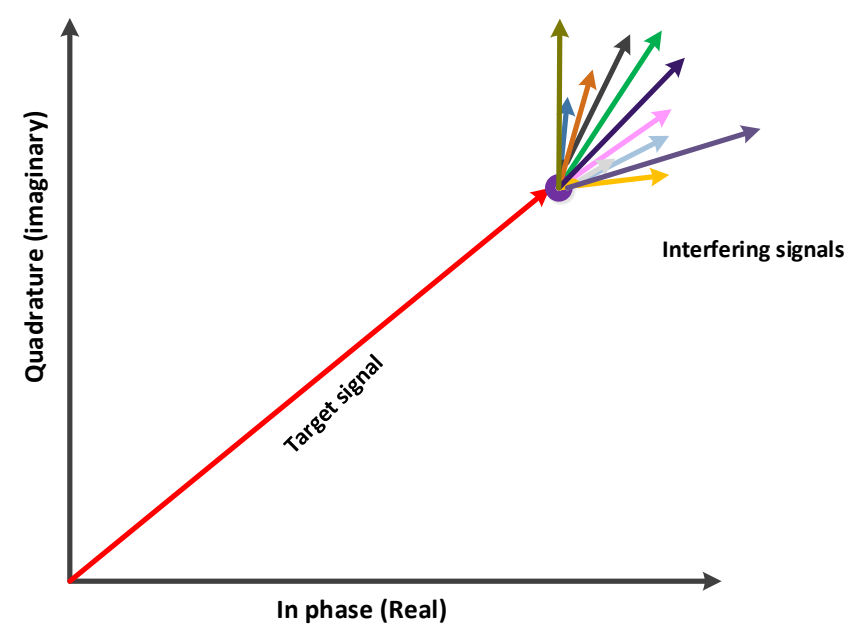

Fig. 6: Interference controlled on symbol by symbol basis to guarantee that the interference is constructive in symbol-level Precoding.

designed such that the difference between the noise free received signal at the receiver(s) and the desired symbol information is minimized in a least squares sense. The constant envelop based techniques exhibit low peak-to-average power ratio (PARP) and their concept presents similar advantages to the one of the directional modulation based transceivers, since ideally they can be also implemented in transceivers of a single RF chain that drives a phase shift network. On the contrary, the involved optimization problems are non-convex due to the constant modulus requirements and thus, they are hard to solve, they support restricted set of constellation points and they treat the interference like the block-level solutions, that is as a harmful component. For now and on we will focus our discussion on the symbol level precoding works.

In symbol-level precoding, the interference can be controlled symbol-by-symbol, so each interfering signal can be rotated to be in the correct detection region as depicted in Fig. 6. The multiuser interference can be classified into constructive or destructive based on whether it facilitates or deteriorates the correct detection of the received symbol (see Fig. 7). A detailed classification of interference is thoroughly discussed in for binary phase shift keying (BPSK) and quadrature phase shift keying (QPSK) in [97] and for $M$-PSK in [210]. The constructive interference pushes the detected constellation point deeper into detection region. Fig. 7 illustrates the two scenario when the interference is destructive and when it is constructive for QPSK modulation. 
To classify the multiuser interference, both the data information and the CSI should be available at the transmitter. the unit-power created interference from the $k^{\text {th }}$ data stream on the $j^{\text {th }}$ user can be formulated as:

$$
\psi_{j k}=\frac{\mathbf{h}_{j}^{T} \mathbf{w}_{k}}{\left\|\mathbf{h}_{j}\right\|\left\|\mathbf{w}_{k}\right\|} .
$$

This metric is used to assess orthogonality among the vectors [50], when $\left|\psi_{j k}\right|$ is close to zero, the vectors are semiorthogonal and when $\left|\psi_{j k}\right|$ is close one , the vectors are co-linear. In block-level precoding, $\left|\psi_{j k}\right|$ should be close to 1 . In symbol-level precoding, not only the magnitude $\left|\psi_{j k}\right|$ is consequential but also its phase $\angle \psi_{j k}$, which plays an important role on deciding whether the interference is constructive (without additional processing) or destructive. The interference can be considered constructive for a certain set of symbols, it can be destructive for a different set given the CSI does not change. An $M$-PSK modulated symbol $s_{j}$, is said to receive constructive interference from another simultaneously transmitted symbol $s_{k}$ which is associated with $\mathbf{w}_{j}$ if and only if the following inequalities hold

$$
\begin{gathered}
\angle s_{j}-\frac{\pi}{M} \leq \arctan \left(\frac{\mathcal{I}\left\{\psi_{j k} s_{k}\right\}}{\mathcal{R}\left\{\psi_{j k} s_{k}\right\}}\right) \leq \angle s_{j}+\frac{\pi}{M}, \\
\mathcal{R}\left\{s_{k}\right\} \cdot \mathcal{R}\left\{\psi_{j k} s_{j}\right\}>0, \mathcal{I}\left\{s_{k}\right\} \cdot \mathcal{I}\left\{\psi_{j k} s_{j}\right\}>0 .
\end{gathered}
$$

The first inequality states the condition on the phase of the interfering signal to be considered constructive without additional processing. For any $M$-PSK modulated symbol, the region of correct detection lies in $\theta_{j} \in\left[\angle s_{j}-\frac{\pi}{M}, \angle s_{j}+\frac{\pi}{M}\right]$, where $\theta_{j}$ is the angle of the detected symbols. In order for the interference to be constructive, the received interfering signal should lie in the region of the target symbol. For the first condition, the $\arctan (\cdot)$ function checks whether the received interfering signal originating from the $s_{k}$ transmit symbol is located in the detection region of the target symbol $s_{j}$. However, trigonometric functions are not one-to-one. This means that it manages to check the two quadrants which the interfering symbol may lie in. To find which one of these quadrants is the correct one, an additional constraint is added to check the sign compatibility of the target and received interfering signals. This was proved in details in [210]. One of the interesting characteristics of the constructive interference between two streams is its mutuality. In more details, if the stream $\mathbf{w}_{j} s_{j}$ constructively interferes with $\mathbf{w}_{k} s_{k}$ (i.e. pushes $s_{k}$ deeper in its detection region), then the interference from transmitting the stream $\mathbf{w}_{k} s_{k}$ is constructive to $s_{j}$ [210]. For constructively interfering symbols, the value of the received signal 

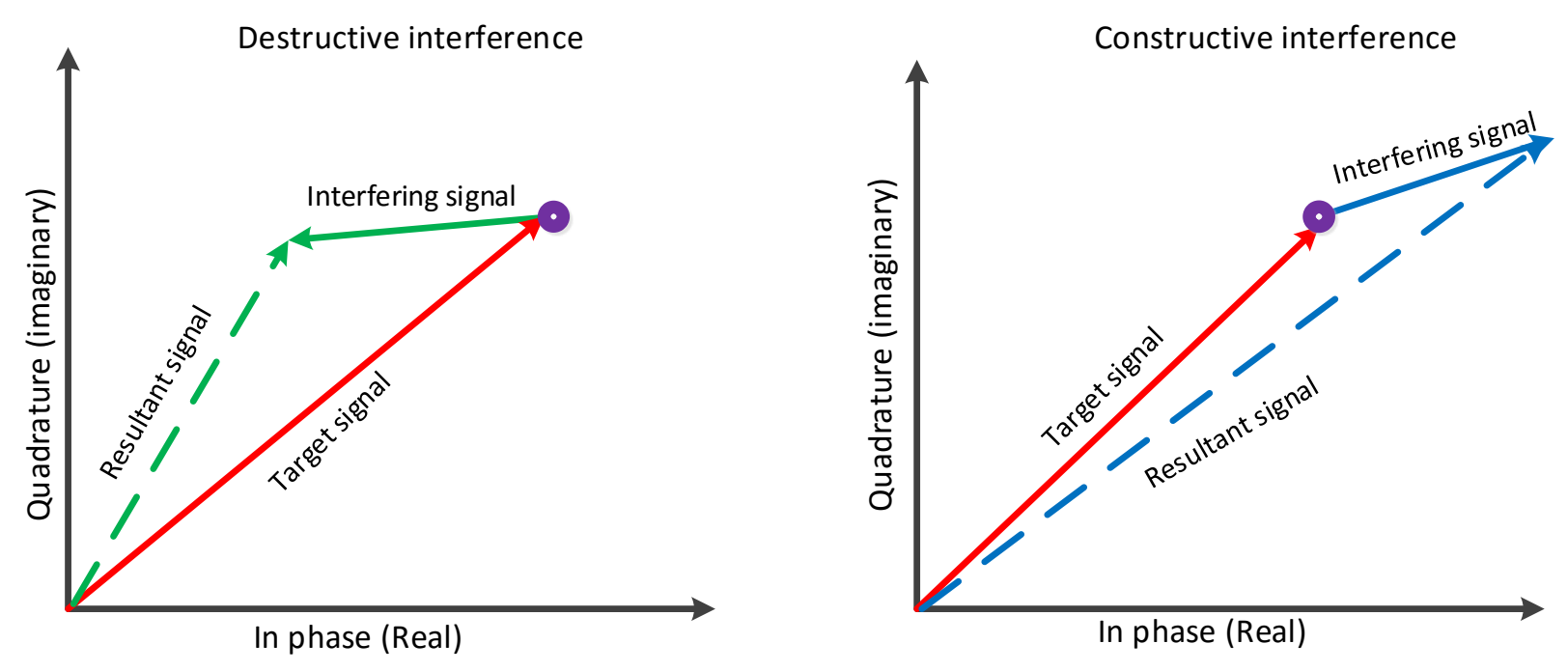

Fig. 7: The first quadrant of QPSK, the adopted scenario is $K=Q=2$. The target signal should be received correctly at the first quadratic. The interference can be destructive as the figure in the left or constructive as the figure in the right, if the interfering signal tries to push the target signal is considered destructive, and if it tries to push it deeper in the correct detection region is considered destructive.

can be bounded as

$$
\left\|\mathbf{h}_{j}\right\| \stackrel{(a)}{\leq}\left|y_{j}\right| \stackrel{(b)}{\leq}\left\|\mathbf{h}_{j}\right\|\left(1+\sum_{\forall k, k \neq j}^{K}\left|\psi_{j k}\right|\right) .
$$

The inequality (a) holds when all simultaneous users are orthogonal (i.e. $\psi_{j k}=0$ ), while (b) holds when all interfering signal are aligned with the transmitted symbol as $\angle s_{k}=\angle \psi_{j k} s_{j}$ and $\psi_{j k}=0, \angle s_{k}=\angle \psi_{j k} s_{j}$, they are constructive without any additional processing. The previous inequality indicates that in the case of constructive interference, having fully correlated signals is beneficial as they contribute to the received signal power. For a generic symbol-level precoding, the previous inequality can be

$$
0 \stackrel{(a)}{\leq}\left|y_{j}\right| \stackrel{(b)}{\leq}\left\|\mathbf{h}_{j}\right\|\left(1+\sum_{\forall k, k \neq j}^{K}\left|\psi_{j k}\right|\right) .
$$

In comparison to block-level precoding techniques, the previous inequality can be reformulated as

$$
0 \stackrel{(a)}{\leq}\left|y_{j}\right| \stackrel{(b)}{\leq}\left\|\mathbf{h}_{j}\right\|
$$




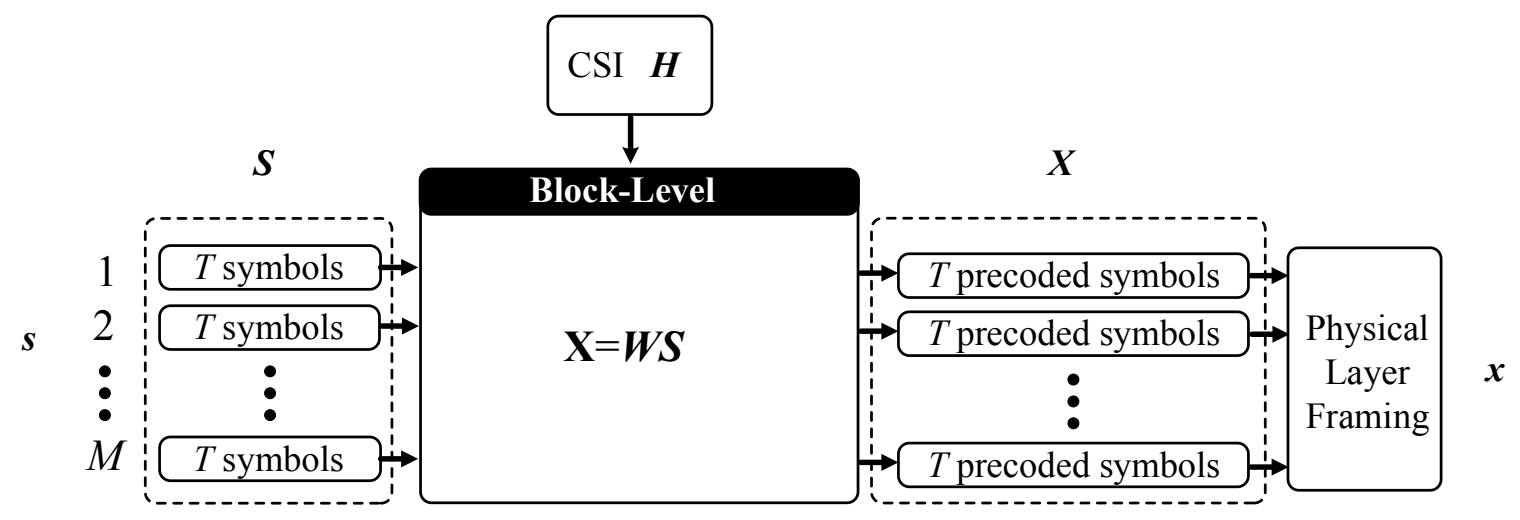

Fig. 8: Schematic Diagram for Block-level Precoding transmitter in which the precoder changes with only CSI.

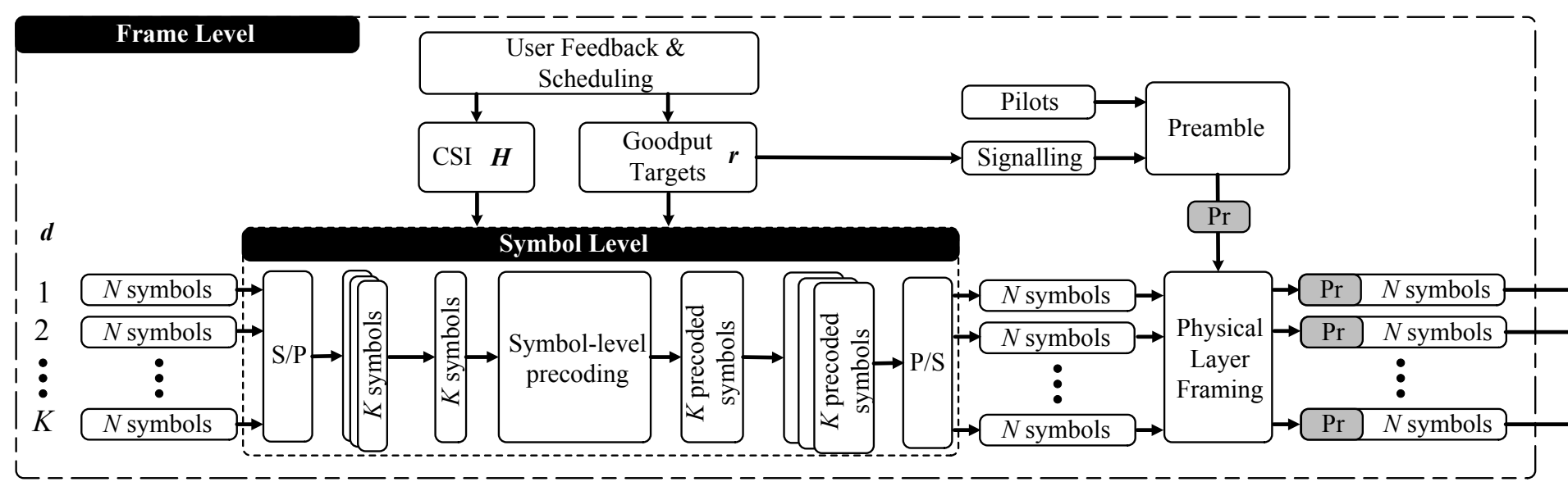

Fig. 9: Schematic Diagram for Symbol-level Precoding transmitter in which the precoder changes with CSI and data symbols.

The worst case scenario can occur when all users are co-linear, that is when $\psi_{j k} \rightarrow 1$. The channel cannot be inverted and thus the interference cannot be mitigated. The optimal scenario takes place when all users have physically orthogonal channels which entails no multiuser interference. Therefore, utilizing CSI and DI leads to higher performance in comparison to employing conventional techniques.

The difference between the block-level and symbol-level precoding techniques is illustrated in Fig. 8-9. Fig. 8 shows how the block-level precoding depends only on the CSI information to optimize $\mathbf{W}$ that carry the data symbols $\mathbf{s}$ and without any design dependency between them. In contrary, symbol-level precoding as illuastrated in Fig. 9 depends on both CSI and the data 
symbol combinations to optimize the precoding matrix $\mathbf{W}$ and the output vector $\mathbf{x}$. The optimal design for symbol-level precoding depends on how to define the optimization problem and more importantly how to define the constructive interference constraints. In [103], [209]-[214], the optimal precoding strategy for the minimization of the total transmit power, whilst guaranteeing QoS targets at each user, was given. For any modulation, the related optimization problem can be written as follows:

$$
\begin{aligned}
\mathbf{w}_{k}(\mathbf{s}, \boldsymbol{\gamma}, \mathbf{H})= & \arg \min _{\mathbf{w}_{k}}\left\|\sum_{k=1}^{K} \mathbf{w}_{k} s_{k}\right\|^{2} \\
\text { s.t. } \quad & \left|\mathbf{h}_{j}^{T} \sum_{k=1}^{K} \mathbf{w}_{k} s_{k}\right|^{2} \unlhd \kappa_{j} \gamma_{j} \sigma^{2}, \forall j \in K \\
& \angle\left(\mathbf{h}_{j}^{T} \sum_{k=1}^{K} \mathbf{w}_{k} s_{k}\right) \unlhd \angle s_{j},
\end{aligned}
$$

where $\unlhd$ is an operator that guarantees that the received signal lies in the correct detection region and $\kappa_{j}=\left|s_{j}\right| / \sqrt{\mathbb{E}_{\mathcal{D}}\left[\left|s_{j}\right|^{2]}\right.}$ denotes short-term factor changes on a symbol-basis and adjusts the long-term SINR based on the amplitude of the desired symbol. By using $\mathbf{x}=\sum_{k=1}^{K} \mathbf{w}_{k} s_{k}$, the previous optimization can be formulated as:

$$
\begin{aligned}
\mathbf{x}(\mathbf{s}, \boldsymbol{\gamma}, \mathbf{H})= & \arg \min _{\mathbf{x}}\|\mathbf{x}\|^{2} \\
\text { s.t } & \left|\mathbf{h}_{j}^{T} \mathbf{x}\right|^{2} \unlhd \kappa_{j} \gamma_{j} \sigma^{2}, \forall j \in K \\
& \angle\left(\mathbf{h}_{j}^{T} \mathbf{x}\right) \unlhd \angle s_{j}, \forall j \in K .
\end{aligned}
$$

For any circular modulation ( $M$-PSK) and amplitude phase shift keying (APSK), the constraints can be formulated as:

$$
\begin{gathered}
\left|\mathbf{h}_{j}^{T} \mathbf{x}\right|^{2} \unlhd \kappa_{j} \gamma_{j} \sigma^{2}, \forall j \in K \\
\angle\left(\mathbf{h}_{j}^{T} \mathbf{x}\right)=\angle s_{j}, \forall j \in K
\end{gathered}
$$

For $M$-PSK, the operator $\unlhd$ can be simplified into $\geq$ and $\kappa_{j}=1, \forall j$. For APSK, the operator $\unlhd$ can be simplified for the outermost constellation point into $\geq$ and for inner constellation points into $=$. The optimization can be tailored to exploit the detection regions for any square multilevel modulation such as quadrature amplitude modulation (QAM), by referring the constraints to 
the in-phase and the quadrature components of the received symbols, rather than their magnitude and phase. In this case, the optimization can be formulated as:

$$
\begin{aligned}
\mathbf{x}(\mathbf{s}, \boldsymbol{\gamma}, \mathbf{H})= & \arg \min _{\mathbf{x}}\|\mathbf{x}\|^{2} \\
\text { s.t } \quad & \mathcal{R}\left\{\mathbf{h}_{j}^{T} \mathbf{x}\right\} \unlhd \sigma \sqrt{\gamma_{j}} \mathcal{R}\left\{s_{j}\right\}, \forall j \in K \\
& \mathcal{I}\left\{\mathbf{h}_{j}^{T} \mathbf{x}\right\} \unlhd \sigma \sqrt{\gamma_{j}} \mathcal{I}\left\{s_{j}\right\}, \forall j \in K
\end{aligned}
$$

where $\mathbf{x} \in \mathbb{C}^{N \times 1}$ is the output vector that modulates the antennas. This problem can be solved efficiently using second order cone programming [229]. It can be connected to broadcast scenario (i.e. physical-layer multicasting [21]), this connection has been thoroughly established and discussed in [103], [210].

Different symbol-level precoding schemes have been proposed in the literature. In [211], [214], the constructive interference precoding design is generalized under the assumption that the received $M$-PSK symbol can reside in a relaxed region in order to be correctly detected. Moreover, a weighted maximization of the minimum SNR among all users is studied taking into account the relaxed detection region. Symbol error rate analysis (SER) for the proposed precoding is discussed to characterize the tradeoff between transmit power reduction and SER increase due to the relaxation. These precoding scheme achieve better energy efficiency in comparison to the technique in [209]-[210]. In [123], a symbol-level precoding scheme aims at manipulating both a desired signal and interfering signals is proposed such that the desired signal can be superimposed with the interfering signals. In this approach, a Jacobian-based algorithm is applied to improve the performance. Furthermore, it has been shown that robustness becomes stronger with an number of co-scheduled users in the systems adopt $M$-PSK modulation.

Since the CSI acquisition in most systems is not perfect, it is important to design symbol-level schemes robust to different types of error. In [122], interference is decomposed into predictable interference, manipulated constructively by a BS, and unpredictable interference, caused by the quantization error. To characterize performance loss by unpredictable interference, the upper bound of the unpredictable interference is derived. To exploit the interference, the BS aligns the predictable interference so that its power is much greater than the derived upper bound. During this process, to intensify the received signal power, the BS simultaneously aligns the predictable interference so that it is constructively superimposed with the desired signal. Different approach of guaranteeing the robustness of the symbol-level precoding is proposed in [122]-[124], [230]. These approaches are based on assuming that the errors in CSI is bounded, and the precoding is 
designed taking into consideration the worst case scenario. The problem in [124] is formulated as second order cone problem and can be solved using conventional convex optimization tools.

Most of the symbol-level precoding literature tackles the symbol-level precoding in single-level modulations (M-PSK) [97]-[101], [123], [124], [220], [230]. In [94], [103], [213], the proposed precoding schemes are generalized to any generic modulation. The relation to physical-layer multicasting is established for any modulation in [103]. A per-antenna consideration is thoroughly discussed in [102]-[94]. In [94], novel strategies based on the minimization of the power peaks amongst the transmitting antennas and the reduction of the instantaneous power imbalances across the different transmitted streams is investigated. These objectives are important due to the per-antenna amplifiers characteristics which results in different amplitude cutoff and phase distortion. As a result, ignoring the previous factors can question the feasibility of employing precoding to multiuser MIMO systems. The work in [94] proposes to design the antenna weights taking into the account the amplifier characteristics by limiting the amount of power variation across the antennas amplifier, which leads to less deviation across the antennas and hence, less distortion.

The applications of symbol-level precoding span different research areas in wireless communications: underlay cognitive radio system [99], [100], [102], [212], coordinated multicell MIMO systems [217], physical-layer security [161], [190], [219], [220] and simultaneous wireless information and power transfer (SWIPT) [221]. For more details about the applications of symbol-level precoding, please look at Table III.

Finally, symbol-level precoding and directional modulation is conceptually the same; since both of them calculate the output of antennas for each symbol combinations. However, both of them have the following main differences: directional modulation is driven by implementational aspects, assuming an analogue architecture with less emphasis on formulating criteria that optimizes the actual precoding weights, that is the reason why we call it analog symbol-level precoding. It also has less emphasis on multiuser and system performance. On the other hand, symbol-level precoding is driven by multiuser performance optimization, taking less consideration into implementation. However, it implicitly assumes a fully digital baseband implementation.

\section{Transmitter architecture}

The main difference between the architecture of symbol-level and block-level architectures is the capability to facilitate the processing symbol by symbol. In Fig.8, the precoding matrix $\mathbf{W}$ is 
designed as a function of $\mathbf{H}$. The data streams for all users are fed through the precoding block, which implements a matrix multiplication step to find the vector that modulates the antennas $\mathbf{x}=$ Ws. In Fig. 9, the data streams are fed to the precoding system to enable the processing. The processing is based on taking the set of simultaneous symbols for all users. Each set can be tackled independently and in parallel fashion in case of no channel memory. The output vector $\mathbf{x}$ is calculated using symbol-level optimization such as (11)-(12) taking the set of symbols and the CSI as inputs. It is stored in a look-up table to use it if the same set of symbols is repeated. The parallel processing can be used to speed up the operation by calculating multiple output vectors simultaneously. The complexity of symbol-level precoding lies in the number of output vector calculations. The advantages of the symbol-level architectures have the potential to simplify the circuit design by removing the multiplication step Ws and directly designing the output vector $\mathrm{x}$.

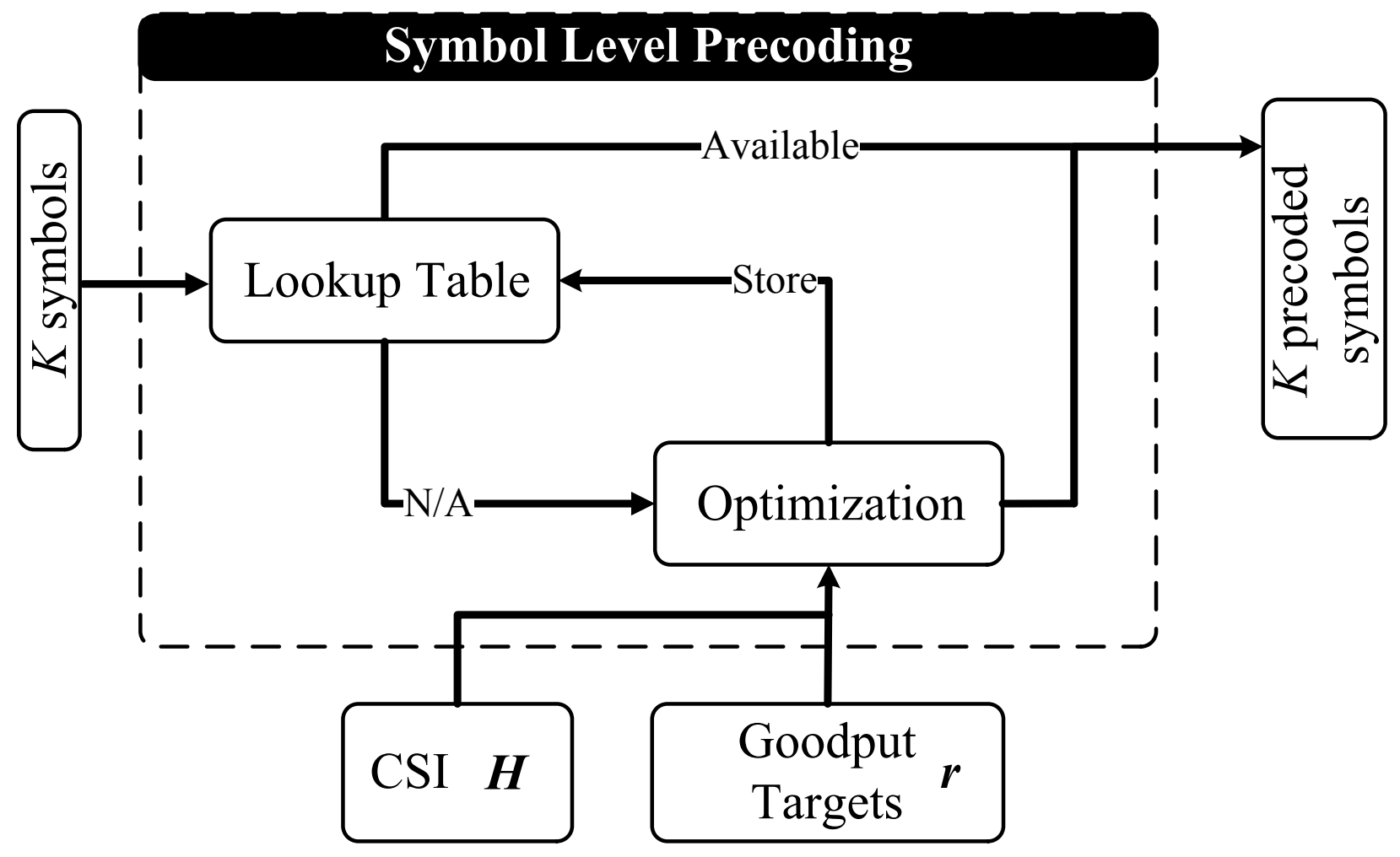

Fig. 10: Detailed block diagram that explains the symbol-level precoding. 


\section{Comparative Study}

In order to assess the relative performance of the precoding techniques discussed in the previous sections, some numerical results are presented in this section. Firstly, the focus is on block-level precoding, both unicast and multicast. Then, the performance of symbol-level precoding is assessed, in comparison to the conventional block-level case.

In the remainder of this section, a system with 4 transmit antennas and 4 users is assumed, hence having $N=K=4$. Moreover, the channel vector of the generic user $j$ is modeled as $\mathbf{h}_{j} \sim \mathcal{C N}\left(0, \sigma_{h}^{2} \mathbf{I}\right)$, with $\sigma_{h}^{2}=1$ and the results are obtained averaging over several channel realizations. Furthermore, we assume a unit AWGN variance for all the users.

\section{A. Block-level Precoding Results}

Considering a unicast framework, Fig. 11 compares the sum-rate performance of ZF precoding, MMSE precoding, and the max-min fair scheme given in (5). A system bandwidth of $250 \mathrm{MHz}$ is assumed for the rate calculation. Interestingly, the best performance is given by MMSE.

Furthermore, Fig. 12 shows how the sum rate is distributed among the users for a specific channel realization. Although the max-min fair approach performs slightly worse than MMSE in terms of sum rate, it is visible how it guarantees a better minimum rate across the users. Therefore, it improves the fairness.

We consider analogous numerical results for comparing the introduced precoding techniques for unicast, multicast, and broadcast (the max-min fair optimization strategy is considered). Fig. 13 displays the sum rate as a function of the total available power. It emerges how the performance improves when different users are grouped so as to receive the same data stream. This can be justified by the fact that in the multicast case the interference is reduced with respect to the unicast case, where each user receives a different stream. The same can be noticed from the result of Fig. 14, where the rate distribution is shown for the three cases considering a specific channel realization.

\section{B. Symbol-level Precoding Results}

In this section, we compare the performance of symbol-level precoding with the equivalent block-level precoding scheme, in a unicast framework. In particular, we consider the power minimization strategy with QoS constraints, given in (4) and in (11) for block-level and symbollevel respectively. A 8-PSK modulation scheme is assumed for the data information. 


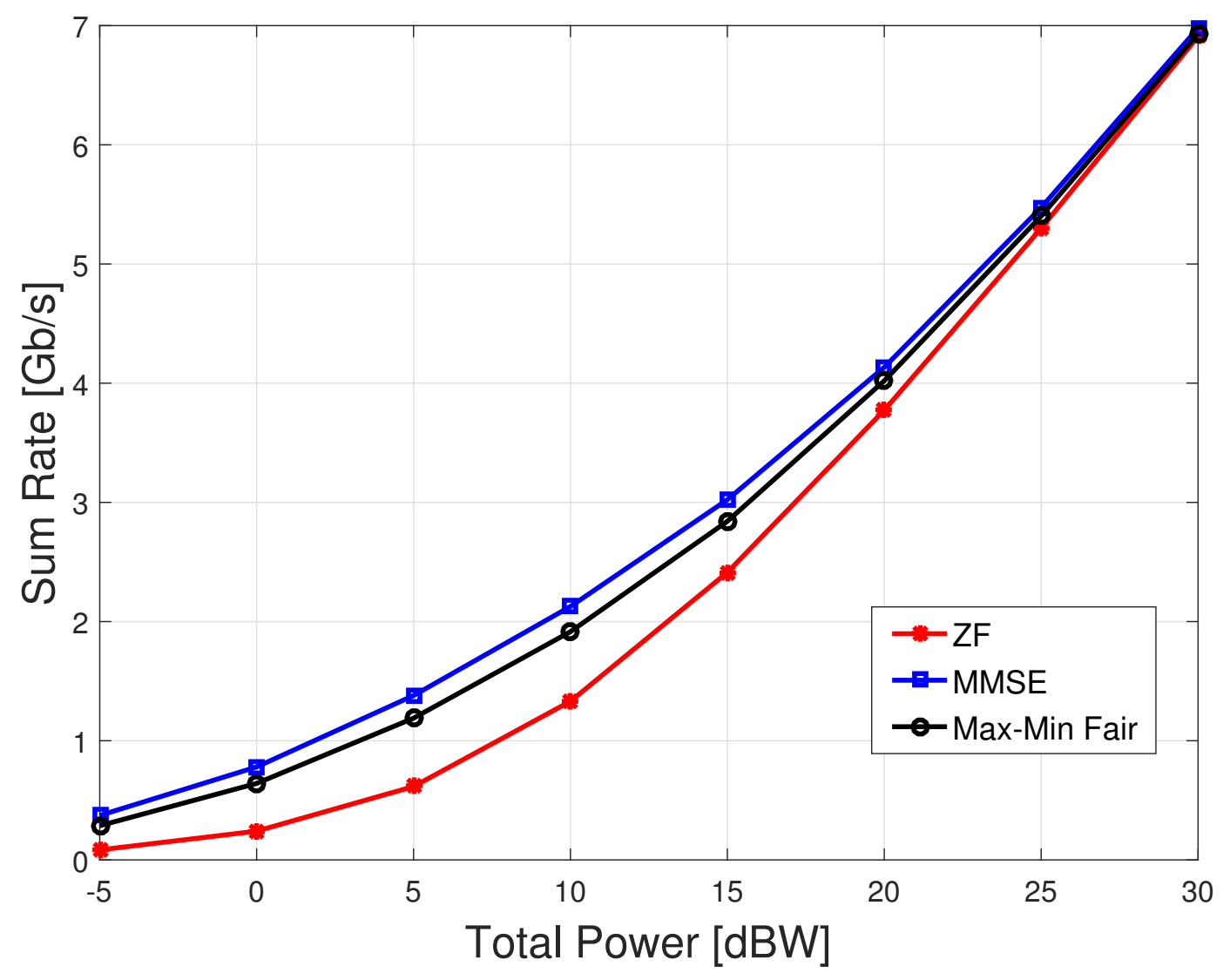

Fig. 11: Sum rate of different unicast block-level precoding, in $\mathrm{Gb} / \mathrm{s}$, versus total available power, in $\mathrm{dBW}$.

Fig. 15 shows the related performance obtained for the two schemes, in terms of attained average SINR, as a function of the required total power. It is clear how the symbol-level precoding scheme outperforms the block-level one in the high SINR regime. This can be justified by considering that this regime, which corresponds to a higher transmitted power, is more interference limited. Accordingly, while the block-level scheme suffers a loss in the SINR, the symbol-level scheme can leverage the interference to improve the overall performance. On the other hand, in the low SINR regime the constructive interference effect does not play a significant role. Actually, in this regime the symbol-level scheme performs even worse than the block-level one: this can be explained by considering that the symbol-level problem (11) is imposing tight equality constraints on the angle of the received symbols, without benefiting of a significant constructive interference. 


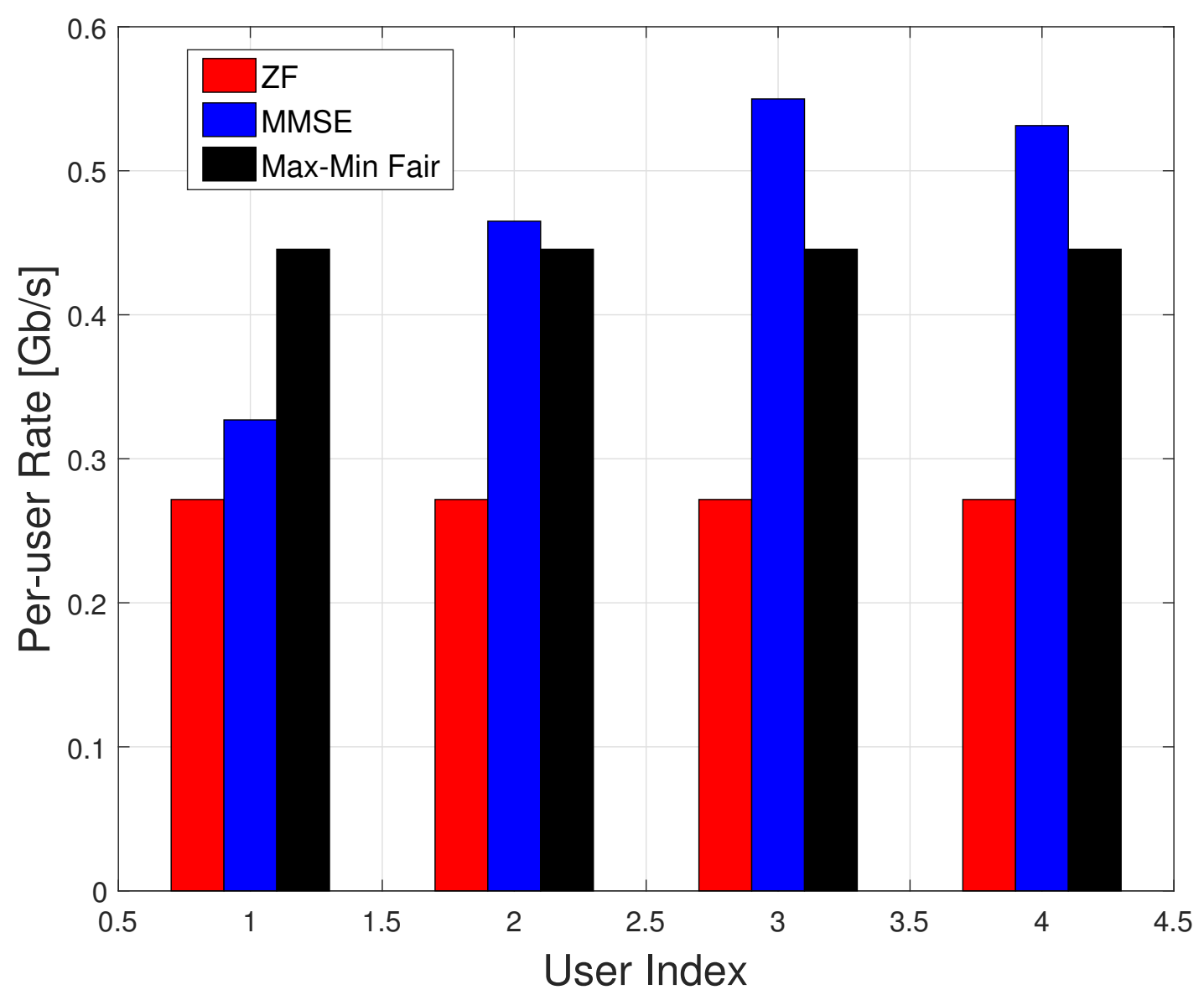

Fig. 12: Per-user rate distribution, in $\mathrm{Gb} / \mathrm{s}$, versus total available power, for a specific channel realization.

Fig. 16 shows an analogous comparative result considering a multi-level modulation. In particular, a 16-QAM modulation scheme is used for the data information. Interestingly, in this case the symbol-level scheme outperforms the block-level one for all available power values, thus also in the low SINR regime. This can be explained by noticing that the symbol-level precoding formulation for rectangular modulations (QAM), provided in (12), imposes more relaxed constraints with respect to the problem for circular modulations ( $M$-PSK, APSK) in (11), because of the different detection regions. In particular, the equality constraints on the symbols' angles do not apply in the case of rectangular modulations. This implies an inherent advantage of the rectangular modulations over the circular ones in the context of the symbol-level precoding formulations of (11), (12). 


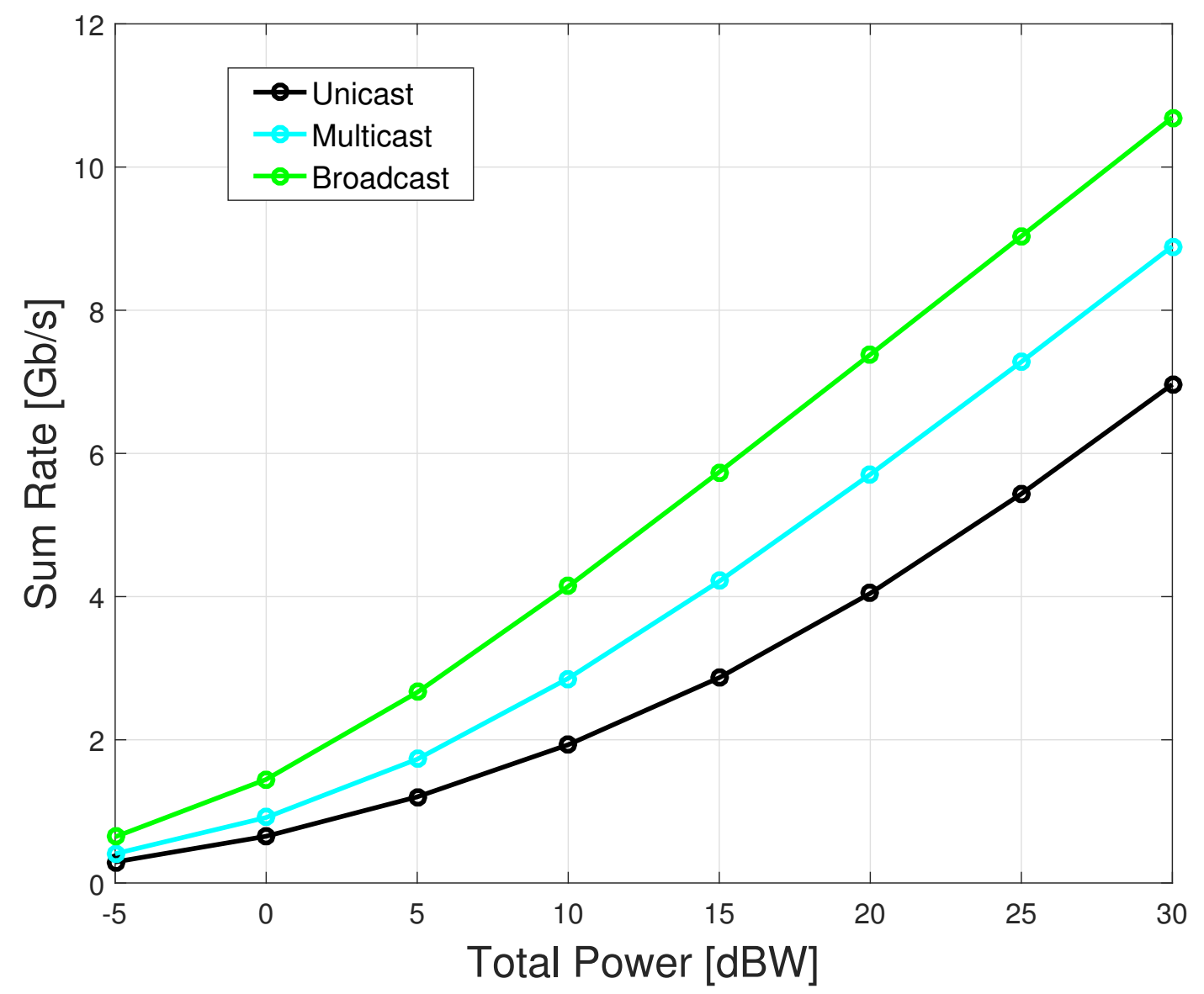

Fig. 13: Sum rate performance of block-level max-min fair for different service types, in $\mathrm{Gb} / \mathrm{s}$, versus total available power, in $\mathrm{dBW}$.

Fig. 17 compares the performance of different modulation techniques 16-QAM, 16-APSK and 16-PSK. It can be concluded that the geometry of modulation has an influential effect on symbol-level precoding systems. The rectangular modulations (QAM) outperforms the circular modulation ( $M$-PSK and APSK) for any power and SINR pair. Apparently, the geometric properties of quadratic modulation make it less restrictive and benefit more from the constructive interference. Although 16-PSK has 16 outer constellation points in comparison with 16-APSK and 16-QAM, it performs the worst since it has more constrained detection regions. This means it is beneficial to have some inner constellation points and more relaxed detection regions for the outer and the outermost constraints. 


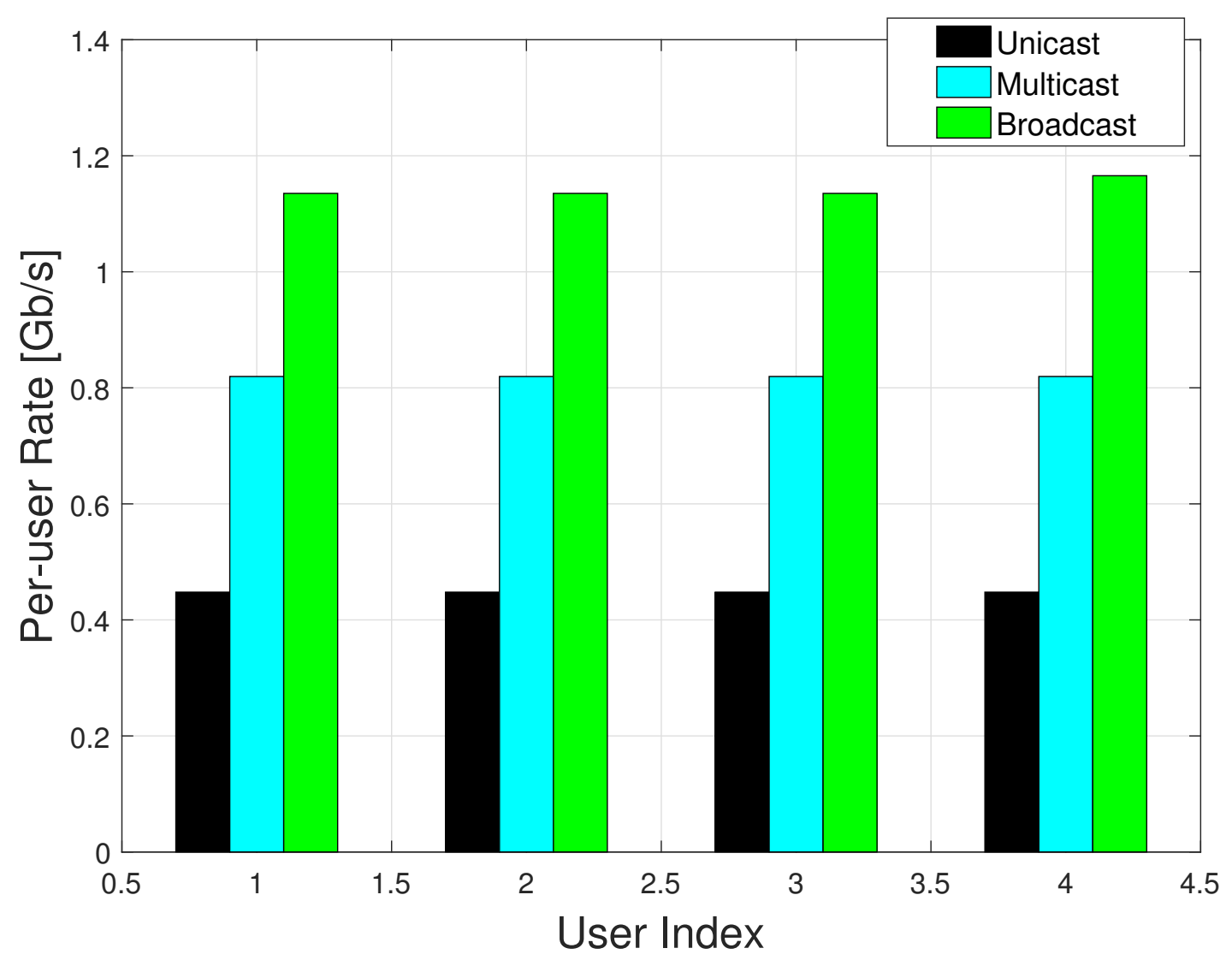

Fig. 14: Per-user rate distribution, in $\mathrm{Gb} / \mathrm{s}$, versus total available power, for a specific channel realization.

\section{Challenges And Open Problems}

\section{A. Robust Precoding}

The accuracy of the estimated CSI plays an important role in designing accurate precoding that can mitigate and exploit the interference created from the simultaneous spatial transmissions. Designing robust precoding strategies is an important topic to tackle, especially when the acquired CSI is not perfect [76], [111]-[114].For these cases, robust precoding under uncertainty is required. In this direction, three robust different designs were proposed in the literature[231]. Namely, the probabilistic design [118], where acceptable performance is guaranteed for some percentage of time, the expectation based design that requires knowledge of the second order channel statistics but cannot guarantee any outage performance [112] and the worst-case design [115]. The latter approach guarantees a minimum QoS requirement for any error realization. 


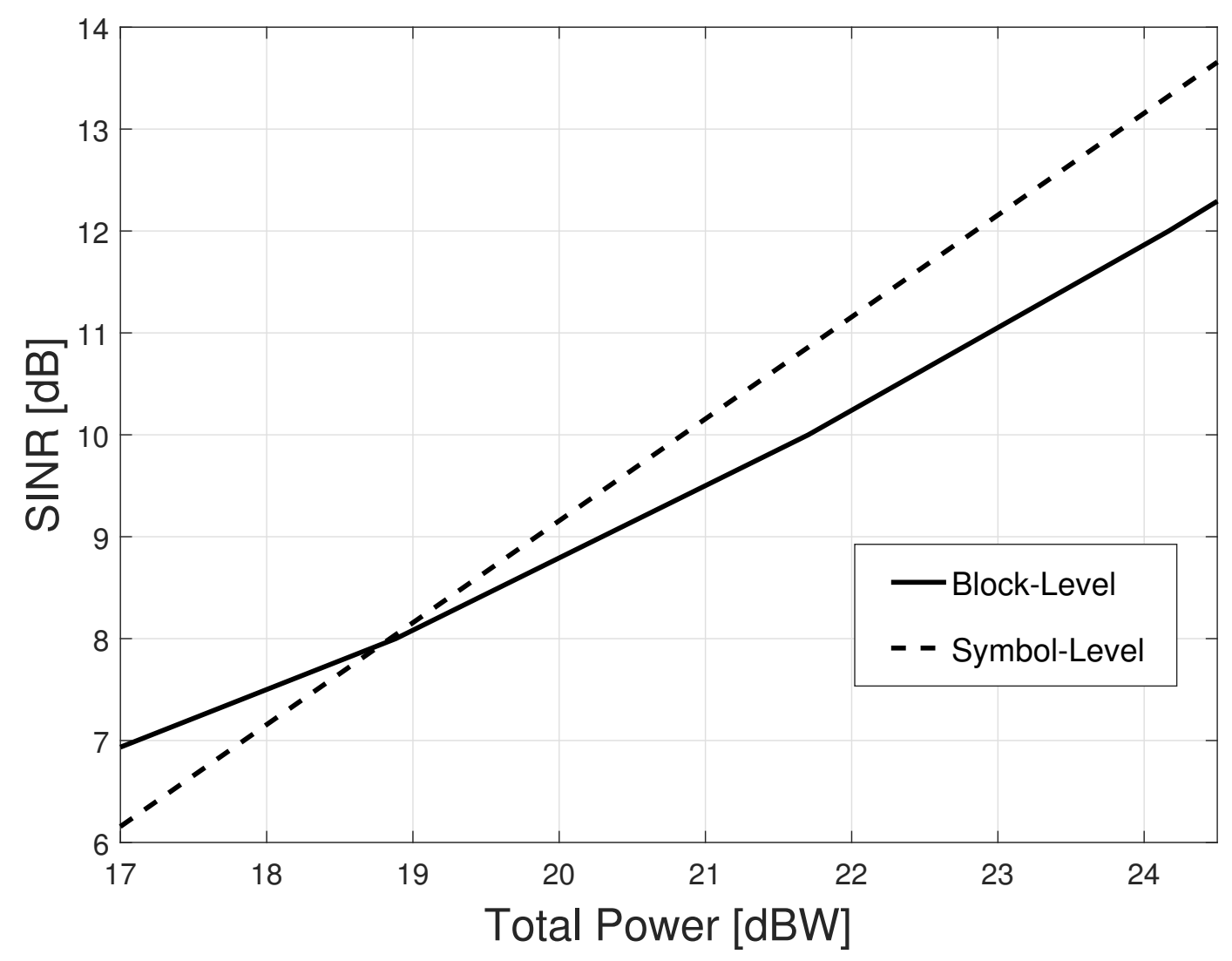

Fig. 15: Average attained SINR of block and symbol-level, in $\mathrm{dB}$, versus total available Power, in $\mathrm{dBW}$, for a 8-PSK modulation Scheme.

Most of the techniques in the literature focus on designing robust strategies for block-level precoding. For symbol-level techniques, there is room to design robust strategies to tackle different types of uncertainties, since the only proposed robust design tackles worst case for $M$-PSK modulations [124]. Robust strategies tackling different kinds of uncertainties for multilevel modulation still need to be addressed to see the full potential of symbol-level precoding.

\section{B. Multiple-antenna Terminals}

Having multiple antenna terminal adds a new dimension that can be utilized in different ways. Most of the literature focuses on exploiting them in unicast block level precoding [195], [232]-[239]. Three methods have been proposed in the literature to use these additional DoF: receive combining [232], multistream multiplexing, and receive antenna selection. All these 


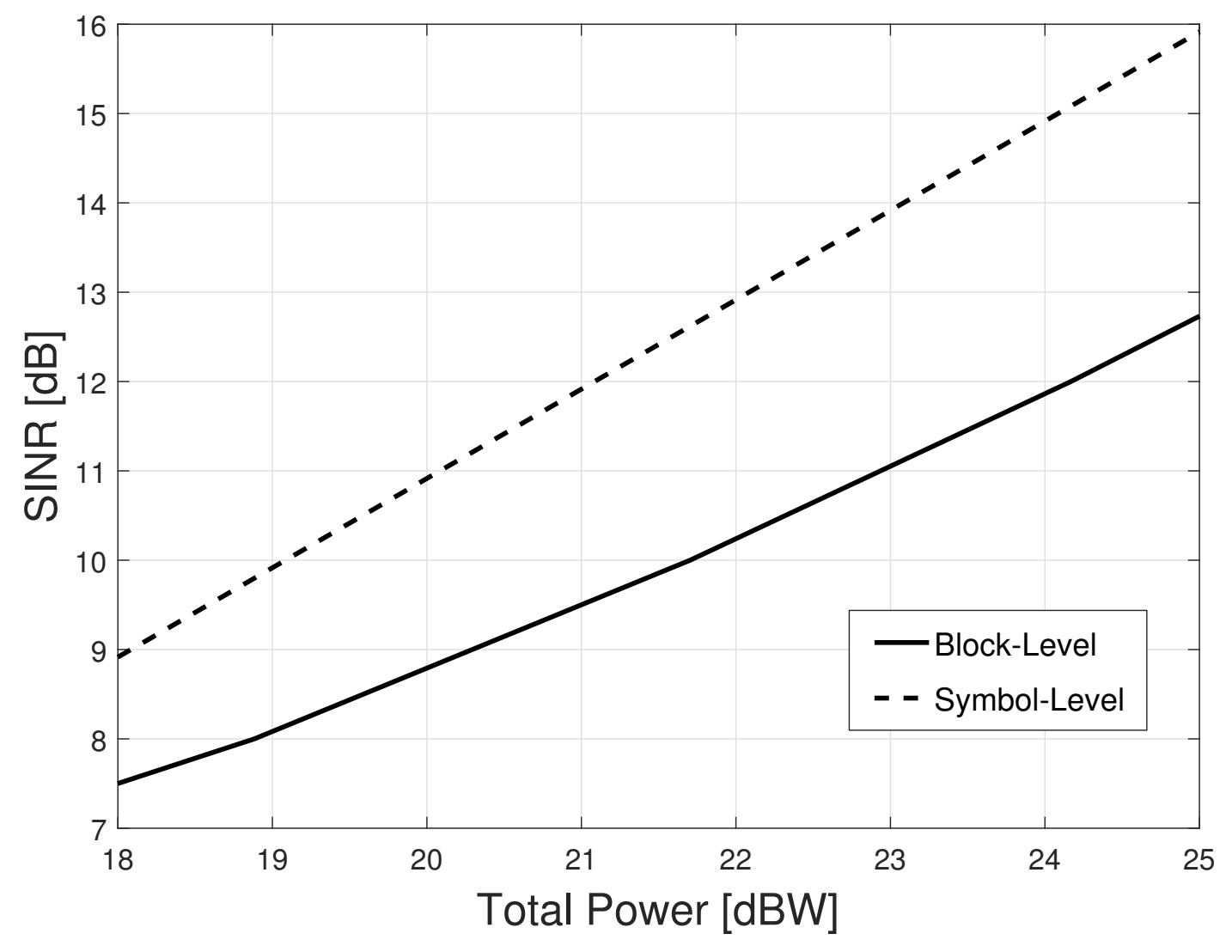

Fig. 16: Average attained SINR of block and symbol-level, in $\mathrm{dB}$, versus total available power, in $\mathrm{dBW}$, for a 16-QAM modulation Scheme.

schemes have their advantages in comparison to single-antenna receiver. In [232], receive antenna combining has been used to reduce channel quantization error in limited feedback MIMO downlink channels, and thus significantly reducing channel feedback requirements. In [233][239], different contradicting conclusions are drawn related to multistream spatial multiplexing. The authors of [233] claim that transmitting at most one stream per user is desirable when there are many users in the system. They justify this statement by using asymptotic results from [236] where $K \rightarrow \infty$. This argumentation ignores some important issues: 1) asymptotic optimality can also be proven with multiple streams per user; 2) the analysis implies an unbounded asymptotic multi-user diversity gain, which is a modeling artifact of fading channels [240]. The diversitymultiplexing tradeff (DMT) brings insight on how many streams should be transmitted in the high-SNR regime [237], [239] considers how a fixed number of streams should be divided among the users. 


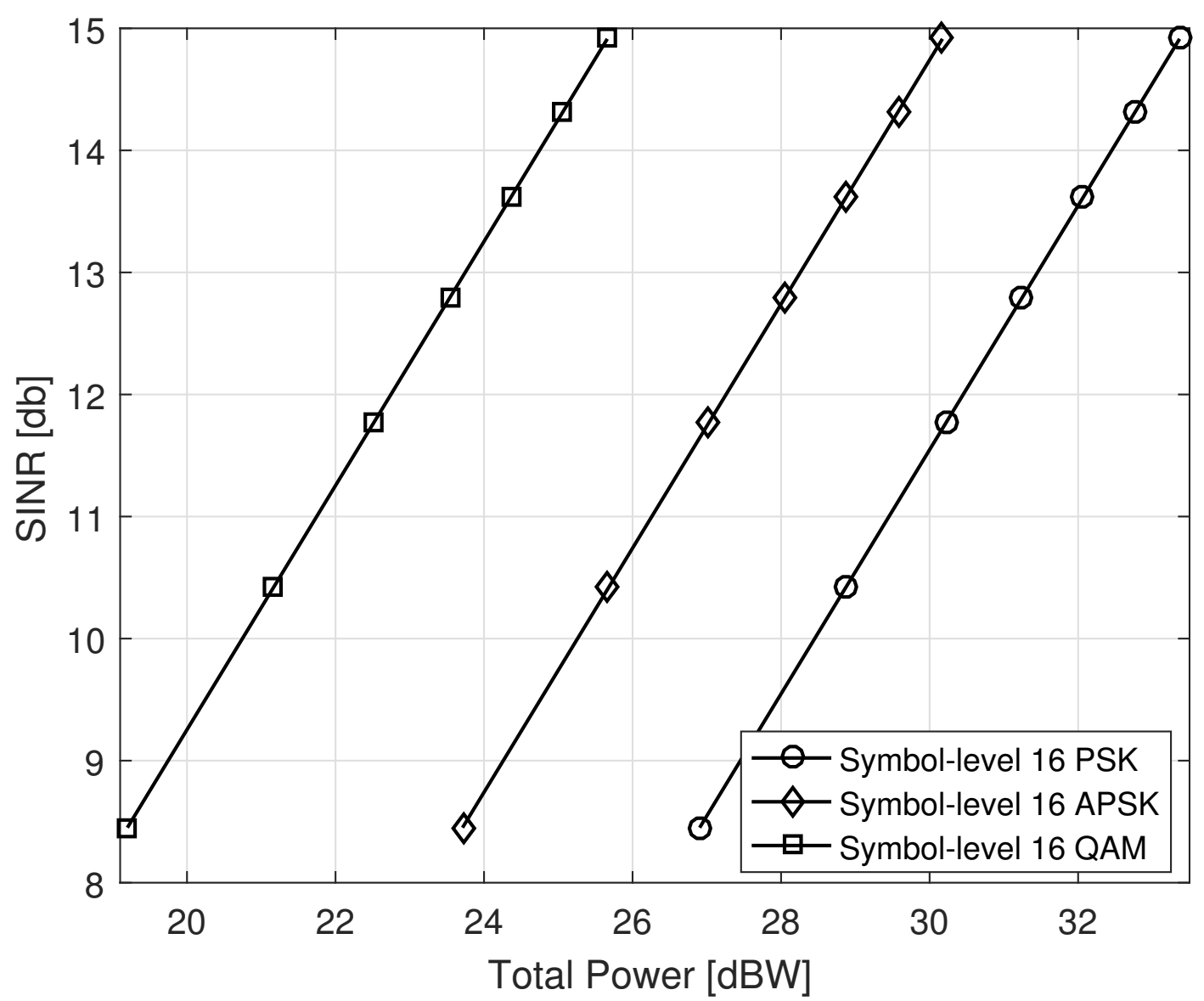

Fig. 17: Average attained SINR of symbol-level, in dB, versus total available Power, in dBW, for 16-PSK, 16-APSK, 16-QAM modulation Schemes.

In the context of this survey, the utilization of multiple antenna at receiver can achieve potential gains and open the doors to new problems that can be solved as discussed below.

1) Symbol-level Precoding: The exploitation of multi-antenna at the receivers has never been discussed for symbol-level precoding. It is interesting to explore the potential gains that can be achieved if we use the different schemes. In [103], simulations have shown that required power to achieve the requested QoS decreases with system size. It is interesting to see how the system will behave if we have multi-stream spatial multiplexing, what is the optimal number of streams per user? Is it modulation dependent? Can the performance show some gains if we have diversity in the system? DMT analysis is required to investigate the system performance at high SNR regime. Moreover, it is worth to see if the different receive antennas selection or receive 
combining algorithms proposed for the unicast block-level precoding are applicable for symbollevel precoding, do we need new algorithms to achieve unprecedented gains in symbol-level precoding.

2) Multicast Precoding: Adding multiple antenna at the receiver can be beneficial for multicast precoding. There is no deep investigation to optimize the multiple antenna at the receiver side. There are still open problems that need to be addressed. Different questions need to be solved: Can developing a new receive combining to optimize the performance be different from blocklevel unicast approaches? Can the user's group affect the optimal receive combining strategy? Can any user belong to more than one group to receive multiple streams simultaneously using multistream spatial multiplexing? In the literature, the optimal group size has been investigated in [241]. DMT analysis is required to study the multigroup multiplexing gains and diversity, and what is the optimal number of the groups and the optimal size of each group with respect to the number of BS 's antennas.

\section{Multicast and Broadcast Symbol-level Precoding}

Symbol-level precoding has been applied so far in unicast precoding to exploit the interference among the different data streams. However, it has the potential to treat inter-group interference in multicast scenarios. The challenge in this direction is to properly exploit the constructive interference when the number of targeted users is larger that the number of transmit antennas. User selection technique has been proposed to enable multigroup multicasting in [242]. The proposed technique aims at grouping the users (to be served) based on their signal and power similarities. The user selection helps in finding the representative channel for each group. This effective channel is selected to guarantee that each user within the group receives the correct information without inducing additional errors and it is used to design the precoding vectors.

The importance of solving of multicasting lies in the framing structure of communications standards, where each frame is received and decoded by all co-group. Therefore, the same precoder should be applied to all users served by the same frame. Since each frame is received and decoded by all co-group users, the design of an optimal frame based precoder is given by solving a multicast multigroup optimization problem. Thus, multicasting allows for an analytically formal modeling of the problem. Therefore, in the context of frame based precoding, the fact that the same precoder needs to apply to the different data of many receivers due to the framing constraint, 
leads to a multicast consideration. The open question here is whether we can find a precoding strategy without using user selection to facilitate the multicasting.

Symbol-level precoding for broadcast scenario in the context of constant envelope precoding has been proposed in [87]. This paper aims at minimizing the maximum SER over all users by jointly optimizing the precoding at the transmitter as well as the constellation rotation and scaling at each of the user receivers. With Special focus on $M$-PSK, it has been shown that this problem is equivalent to a non-convex QCQP, for which an approximate solution can be found via an efficient SDR based algorithm.

\section{Symbol-level Precoding Side-Effects on other Blocks of the Communication Chain}

1) Precoded Pilots for SNR Estimation in Symbol-level Precoding: These functions are often neglected in precoding studies, but they are crucial in implementing a novel precoding method.

Focusing on SNR estimation, this presents a challenge when symbol-level precoding is used. The reason is that unless per-user SNR constraints are imposed the instantaneous received power at each user ranges depending on the input symbol vector. In general, the block-level SNR can be estimated by averaging over a large number precoded input symbol vectors. However, due to the pilot overhead the number of input symbol vectors that can be utilized is limited. The challenge here is to devise pilot design techniques that can reliably estimate the average SNR with a limited pilot length.

2) Modulation and Coding Allocation: Focusing on modulation and code allocation and scheduling, these are important functions which raise cross-layer issues between the physical, MAC and network layers. More specifically, the modulation and coding allocation delimits the achieved rates at each user. The modulation and coding is assigned per user based on the predicted average SNR over a symbol block. In conventional block-level precoding, this average SNR can be efficiently calculated at the transmitter given the scheduled set of users. However, in symbol-level precoding the calculation is more complex since it has to be calculated symbol-bysymbol and averaged over a statistically important set of symbol vectors. Computational-efficient heuristic methods for this process is an important open topic. It should be noted that a workaround is for the users to feedback the requested rates to the transmitter as suggested in [103]. 


\section{E. Massive MIMO}

Massive MIMO (also known as Large-Scale Antenna Systems) is an emerging technology, that scales up MIMO by possibly orders of magnitude to utilize the huge spatial multiplexing gains [91], [126], [137], [137]-[140], [143], [155], [205], [206], [224], [243]-[249]. The basic premise behind massive MIMO is to glean all the benefits of conventional MIMO, but on a much greater scale. The anticipated huge spatial multiplexing gains (degrees-of-freedom DoF) are achieved by coherent processing over large antenna arrays, which result in strong signal gains, low interference, reduced latency, and robustness to imperfect channel knowledge. This comes at the expense of infrastructure costs; the hardware requirements and circuit power consumption scale linearly with the number of BS antennas $N$. In contrast to the current systems, with conventional expensive and power-hungry BS antenna circuits, the main key to cost-efficient deployment of large arrays is low-cost antenna circuits with low power consumption. The challenge is to make many low-precision components work effectively together. Such low-cost transceivers are prone to hardware imperfections, but it has been conjectured that the huge degrees-of-freedom would bring robustness to such imperfections. Another challenge in massive MIMO systems is the CSI acquisition [250]-[255]. In the literature, different acquisition techniques in time division duplexing (TDD) and frequency division duplexing (FDD) are proposed. The applications of symbol-level and multicast precoding to massive MIMO are discussed below:

1) Symbol-level Precoding: In the context of this paper, symbol-level precoding can be a good candidate to be utilized in massive MIMO system. The premise of having a transmitter equipped with many more antennas than the number of served users can produce an excess of degrees of freedom. These additional DoFs could be potentially exploited in symbol-level precoding to improve the conventional performance metrics, but also to further shape desirable waveform properties such as peak to average power ratio (PAPR) and spectral characteristics. This opens the doors to a very promising direction, since it might entail more cost-efficient and less complex transmitter architectures. Moreover, the impact of having limited CSI on the waveform design for massive MIMO is still an open problem that needs to be addressed.

2) Multicast Precoding: In multicast precoding, a common assumption is that the number of served users is higher than the number of transmit antennas. However, the premise of massive MIMO can overcome this assumption and enable a different view of multicast precoding [42], [91], [143]. When the number of transmit antennas is abundant, we might be able to enable multirank transmission to each group to serve efficiently more users, especially when they belong to 
the same group but they have semi-orthogonal channels.

\section{F. Millimeter Wave}

The spectrum congestion in frequencies that are already occupied for mobile services along with the enormously increasing demands for mobile services, forces the wireless communications industry to explore systems adapted to frequencies within the so-called millimeter Wave (mmWave) band [256], [257]. The development of such mmWave transceivers is a very challenging task. Due to their nature, mmWave signals suffer from severe degradations though, due to the short wavelength of mmWave frequencies, a prospect transceiver may employ large array structures for providing high beamforming gains or improvements in the systems spectral efficiency via precoding techniques. Existing digital pre/post-coding techniques, developed in the past years for lower frequency MIMO systems are not suitable for systems of large antenna arrays due to high demands in hardware complexity and power consumption. This is the case since a fully digital transceiver requires a dedicated Radio Frequency (RF) chain per antenna which includes a number of different electronic elements (e.g., Digital-to-Analog/Analog-toDigital converters) that are of high hardware complexity and power consumption, especially for large antenna arrays.

Thus, recent literature approaches seek for solutions that are based on transceivers employing only few number of RF chains compared to the number of antennas and apply hybrid analogdigital precoding to optimize the transmission [131], [132]. The latter techniques are based on a two stage precoder, a digital one applied in the baseband domain and an analog one, applied in the RF domain via a network of phase shifters. While a number of different works [71], [134], [135], [207] were developed in the past in the context of hybrid precoding with satisfactory performance, it is possible that in several cases their implementation may still be of high complexity and power consumption [133]. From that point of view, it is highly desirable to reduce the complexity and power consumption as much as possible, that is used by transceivers of a single RF chain, e.g. by removing completely the digital counterpart of the hybrid precoder. Unfortunately, such a RFonly beamformer can support only single stream and very primitive multi-user communications resulting in severe performance losses. This is the point were directional modulation aims at stepping in to provide efficient precoding schemes for single RF chain transceivers to support multiple streams. 
From the discussion given in Section IV.A, directional modulation techniques develop symbol level precoding directly in the RF domain via digitally controlled analog components (e.g. phase shifters and attenuators). However, there are several challenges toward the implementation of a fully functional and efficient transceiver related to the impairments on the analog hardware that could result in severe performance degradation, efficient CSI estimation techniques, since there is no straightforward way to estimate the required information and waveform design aspects.

Furthermore, as it was discussed in Section III.A, broadcast precoding techniques were recently examined for hybrid analog digital transceivers [70], [71], [84]. Due to the increasing interest around hybrid or in general solutions that exhibit low complexity in large array systems, such as the mmWave or massive MIMO ones, several digital communications techniques have to re-examined in order to propose solutions that can be applied efficiently in the latter systems. Thus, the numerous digital techniques developed in the context of broadcast precoding during the past years, could be examined towards that direction providing new challenges and rekindle the interest in this well-studied field.

\section{G. Connection with Standards}

In this section, a short discussion regarding the connection of the proposed methods to the standards will be given. It is noteworthy to mention that precoding in the sense of MU-MIMO is already included in the several known standards. For example in 2010, the LTE8 TM5 Codebook based-Precoding was introduced at the well known Long Term Evolution (LTE) standard [258] and the VDSL2 Vectoring for self FEXT was introduced in the Very-high-bit-rate digital subscriber line (VDSL) one [259]. In 2014 the Wi-Fi IEEE802.11ac was introduced in the Wi-Fi standard [260]. Furthermore, in 2015, the DVBS2X Superframing is introduced in DVBS2X standard [261]. Finally, it is expected by 2020 the MU-MIMO to be introduced also in the 5G standard, such that MU-Precoding is supported for mm-Wave and Massive MIMO techniques. As a final point, we should highlight that the exact precoding algorithm is often not standardized and is left as a means of competition among equipment manufacturers. In other words, the presented algorithmic solutions in the present tutorial may be adopted by any system that supports the MU-MIMO functionality.

\section{CONCLusions}

In the era of heterogeneous networks, there are many challenges to overcome in the context of multiuser MIMO to achieve better resource utilization. 
In this context, this survey classified the multiuser MIMO precoding strategies with respect to two major axes: the number of users addressed by each information stream, and the switching rate of the precoding coefficients. According to the first classification criterion, unicast, multicast, and broadcast precoding strategies have been throughly discussed. To achieve the optimal network efficiency (throughput, energy efficiency, delay, ...etc), an optimized combination of these transmission strategies can be the new interface for the next wireless generation.

With respect to the second classification criterion, i.e., the switching rate, block-level precoding and symbol-level precoding schemes have been considered. While the former class refers to the conventional schemes, whereas precoding exploits the CSI and is applied over symbol blocks, the latter class refers to novel techniques applying precoding on a symbol-by-symbol basis, thus able to exploit the data information, together with the CSI, in the signal design. We introduced directional modulation and symbol-level precoding for constructive interference where they share the same conceptual model, designing the antenna weights on symbol by symbol basis. However, the directional modulation focuses on the implementational aspects of the concept while the symbol-level precoding focuses on the multiuser optimization aspect. Some representative optimization strategies for symbol-level precoding have been discussed, both for single-level modulation ${ }^{8}$ and multi-level modulation schemes ${ }^{9}$. Despite the fact that symbollevel precoding techniques seem to be futuristic since they incur huge computational complexity at the base station, it can be argued that computational complexity can be transferred to the cloud RAN level [262].

In order to assess the performance of the presented precoding schemes, some numerical results have been presented in a comparative fashion, in terms of attained rate and SINR at the receivers' side. In the context of block-level precoding, the results highlight how the optimization-based schemes outperform the closed-form solutions with respect to specific targeted objectives, e.g., the fairness amongst the users. Moreover, it emerged how, by applying the proper precoding schemes, the multicast framework can show better performance than the unicast one, given a fixed total number of users. Furthermore, it has been shown how symbol-level precoding outperforms the corresponding block-level scheme in interference limited regimes. Based on this observation, the interference in future networks can be exploited provided that we can

\footnotetext{
${ }^{8}$ The term "constant-energy" can be used to refer $M$-PSK modulation

${ }^{9}$ The term "non-constant" energy can be used to refer MQAM and APSK modulations
} 
afford the burden of the computational complexity. Symbol-level precoding schemes do not need complicated physical architectures which make them good candidates to be employed especially with emerging technologies such as massive MIMO and millimeter wave.

\section{REFERENCES}

[1] R. H. Roy III and B. Ottersten, “Spatial division multiple access wireless communication systems,” USA Patent 5,515,378, 1991.

[2] E. Björnson, M. Bengtsson, and B. Ottersten, "Optimal multiuser transmit beamforming: A difficult problem with a simple solution structure [lecture notes],” IEEE Signal Processing Magazine, vol. 31, no. 4, pp. 142-148, Jul 2014.

[3] D. Gesbert, M. Kountouris, R. Heath Jr., C. byoung Chae, and T. Salzer, "Shifting the MIMO paradigm," IEEE Signal Processing Magazine, vol. 24, no. 5, pp. 36-46, Sep 2007.

[4] E. Castaneda, A. Silva, A. Gameiro, and M. Kountouris, "An overview on resource allocation techniques for multi-user MIMO systems," IEEE Communications Surveys \& Tutorials, pp. 1-1, 2016.

[5] Q. Li, G. Li, W. Lee, M. il Lee, D. Mazzarese, B. Clerckx, and Z. Li, "MIMO techniques in WiMAX and LTE: a feature overview," IEEE Communications Magazine, vol. 48, no. 5, pp. 86-92, May 2010.

[6] P. Zetterberg and B. Ottersten, "The spectrum efficiency of a base station antenna array system for spatially selective transmission," IEEE Transactions on Vehicular Technology, vol. 44, no. 3, pp. 651-660, 1995.

[7] D. Gesbert, S. Hanly, H. Huang, S. S. Shitz, O. Simeone, and W. Yu, "Multi-cell MIMO cooperative networks: A new look at interference," IEEE Journal on Selected Areas in Communications, vol. 28, no. 9, pp. 1380-1408, Dec 2010.

[8] S. Bassoy, H. Farooq, M. A. Imran, and A. Imran, "Coordinated multi-point clustering schemes: A survey," IEEE Communications Surveys \& Tutorials, vol. 19, no. 2, pp. 743 - 764, Secondquarter 2017.

[9] S. Schwarz and M. Rupp, "Exploring coordinated multipoint beamforming strategies for 5G cellular," IEEE Access, vol. 2, pp. 930-946, 2014.

[10] G. Y. Li, J. Niu, D. Lee, J. Fan, and Y. Fu, “Multi-cell coordinated scheduling and MIMO in LTE,” IEEE Communications Surveys \& Tutorials, vol. 16, no. 2, pp. 761-775, 2014.

[11] P. Marsch and G. P. Fettweis, Coordinated Multi-Point in Mobile Communications: From Theory to Practice. Cambridge University Press, 2011.

[12] P.-D. Arapoglou, K. Liolis, M. Bertinelli, A. Panagopoulos, P. Cottis, and R. D. Gaudenzi, "MIMO over satellite: A review," IEEE Communications Surveys \& Tutorials, vol. 13, no. 1, pp. 27-51, 2011.

[13] M. A. Vazquez, A. Perez-Neira, D. Christopoulos, S. Chatzinotas, B. Ottersten, P.-D. Arapoglou, A. Ginesi, and G. Tarocco, "Precoding in multibeam satellite communications: Present and future challenges," IEEE Wireless Communications, vol. 23, no. 6, pp. 88-95, Dec 2016.

[14] P.-D. Arapoglou, A. Ginesi, S. Cioni, S. Erl, F. Clazzer, S. Andrenacci, and A. Vanelli-Coralli, "DVB-S2X-enabled precoding for high throughput satellite systems," International Journal of Satellite Communications and Networking, vol. 34, no. 3, pp. 439-455, Jun 2015.

[15] A. R. Forouzan, M. Moonen, J. Maes, and M. Guenach, "Joint level 2 and 3 dynamic spectrum management for downstream DSL," IEEE Transactions on Communications, vol. 60, no. 10, pp. 3111-3122, Oct 2012.

[16] P. P. L. T. Berger, A. Schwager and D. Schneider, MIMO Power Line Communications, EMC and C. P. Advanced Processing, Eds. Taylor \& Francis Inc, 2013.

[17] L. T. Berger, A. Schwager, P. Pagani, and D. M. Schneider, "MIMO power line communications," IEEE Communications Surveys \& Tutorials, vol. 17, no. 1, pp. 106-124, 2015. 
[18] Q. Wang, Z. Wang, and L. Dai, "Multiuser MIMO-OFDM for visible light communications," IEEE Photonics Journal, vol. 7, no. 6, pp. 1-11, Dec 2015.

[19] H. Shen, Y. Deng, W. Xu, and C. Zhao, "Rate-maximized zero-forcing beamforming for VLC multiuser MISO downlinks," IEEE Photonics Journal, vol. 8, no. 1, pp. 1-13, Feb 2016.

[20] K. Cai, M. Jiang, and X. Ma, "Photodetector selection aided multiuser MIMO optical OFDM imaging visible light communication system," IEEE Access, vol. 4, pp. 9870-9879, 2016.

[21] N. Sidiropoulos, T. Davidson, and Z.-Q. Luo, "Transmit beamforming for physical-layer multicasting," IEEE Transactions on Signal Processing, vol. 54, no. 6, pp. 2239-2251, Jun 2006.

[22] N. Jindal and Z. quan Luo, "Capacity limits of multiple antenna multicast," in 2006 IEEE International Symposium on Information Theory, Jul 2006.

[23] M.-C. Yue, S. X. Wu, and A. M.-C. So, "A robust design for MISO physical-layer multicasting over line-of-sight channels," IEEE Signal Processing Letters, vol. 23, no. 7, pp. 939 - 943, July 2016.

[24] A. Abdelkader, A. B. Gershman, and N. D. Sidiropoulos, "Multiple-antenna multicasting using channel orthogonalization and local refinement," IEEE Transactions on Signal Processing, vol. 58, no. 7, pp. 3922 - 3927, 2010.

[25] B. Gopalakrishnan and N. D. Sidiropoulos, "High performance adaptive algorithms for single-group multicast beamforming," IEEE Transactions on Signal Processing, vol. 63, no. 16, pp. 4373 - 4384, August 2015.

[26] V. Ntranos, N. D. Sidiropoulos, and L. Tassiulas, “On multicast beamforming for minimum outage," IEEE Transactions on Wireless Communications, vol. 8, no. 6, pp. 1536-1276, June 2009.

[27] I. H. Kim, D. J. Love, and S. Y. Park, "Optimal and successive approaches to signal design for multiple antenna physical layer multicasting," IEEE Transactions on Communications, vol. 59, no. 8, pp. 2316 - 2327, 2011.

[28] X. Xu, B. Du, and C. Wang, “On the bottleneck users for multiple-antenna physical-layer multicasting," IEEE Transactions on Vehicular Technology, vol. 63, no. 6, pp. 2977 - 2982, 2014.

[29] G. Caire and S. Shamai, "On the achievable throughput of a multiantenna Gaussian broadcast channel," IEEE Transactions on Information Theory, vol. 49, no. 7, pp. 1691-1706, Jul 2003.

[30] E. Karipidis, N. Sidiropoulos, and Z.-Q. Luo, "Transmit beamforming to multiple co-channel multicast groups," in IEEE International Workshop on Computational Advances in Multi-Sensor Adaptive Processing, 2005.

[31] E. Karipidis, N. D. Sidiropoulos, and Z.-Q. Luo, "Far-field multicast beamforming for uniform linear antenna arrays," IEEE Transactions on Signal Processing, vol. 55, no. 10, pp. 4916-4927, Oct 2007.

[32] E. Karipidis, N. Sidiropoulos, and Z.-Q. Luo, "Quality of service and max-min fair transmit beamforming to multiple cochannel multicast groups," IEEE Transactions on Signal Processing, vol. 56, no. 3, pp. 1268-1279, Mar 2008.

[33] D. Christopoulos, S. Chatzinotas, and B. Ottersten, "Multicast multigroup precoding and user scheduling for frame-based satellite communications," IEEE Transactions on Wireless Communications, vol. 14, no. 9, pp. 4695-4707, Sep 2015.

[34] _ - "Multicast multigroup beamforming under per-antenna power constraints," in 2014 IEEE International Conference on Communications (ICC), Jun 2014.

[35] — - "Weighted fair multicast multigroup beamforming under per-antenna power constraints," IEEE Transactions on Signal Processing, vol. 62, no. 19, pp. 5132-5142, Oct 2014.

[36] _ - "Multicast multigroup beamforming for per-antenna power constrained large-scale arrays," in 2015 IEEE 16th International Workshop on Signal Processing Advances in Wireless Communications (SPAWC), Jun 2015.

[37] V. Joroughi, M. Ángel Vàzquez, and A. I. Prez-Neira, "Generalized multicast multibeam precoding for satellite communications," IEEE Transactions on Wireless Communications, vol. 16, no. 2, pp. 952 - 966, February 2017.

[38] Y. Gao and M. Schubert, "Group-oriented beamforming for multi-stream multicasting based on quality-of-service 
requirements," in Computational Advances in Multi-Sensor Adaptive Processing, 2005 1st IEEE International Workshop on. IEEE, 2005, pp. 193-196.

[39] Y. Silva and A. Klein, "Linear transmit beamforming techniques for the multigroup multicast scenario," IEEE Transactions on Vehicular Technology, vol. 58, no. 8, pp. 4353-4367, Oct 2009.

[40] K. L. Law, I. Wajid, and M. Pesavento, "Robust downlink beamforming in multi-group multicasting using trace bounds on the covariance mismatches," in 2012 IEEE International Conference on Acoustics, Speech and Signal Processing (ICASSP), Mar 2012.

[41] S. X. Wu, Q. Li, M.-C. So, and W.-K. Ma, "A stochastic beamformed amplify-and-forward scheme in a multigroup multicast MIMO relay network with per-antenna power constraints," IEEE Transactions on Wireless Communications, pp. 1-1, 2016.

[42] M. Sadeghi, L. Sanguinetti, R. Couillet, and C. Yuen, "Reducing the computational complexity of multicasting in largescale antenna systems," submitted, 2017.

[43] Y. Wu, M. Wang, C. Xiao, Z. Ding, and X. Gao, "Linear precoding for MIMO broadcast channels with finite-alphabet constraints," IEEE Transactions on Wireless Communications, pp. 1-15, 2012.

[44] A. Lozano, A. Tulino, and S. Verdu, "Optimum power allocation for parallel gaussian channels with arbitrary input distributions," IEEE Transactions on Information Theory, vol. 52, no. 7, pp. 3033-3051, Jul 2006.

[45] F. Perez-Cruz, M. R. D. Rodrigues, and S. Verdu, "MIMO gaussian channels with arbitrary inputs: Optimal precoding and power allocation," IEEE Transactions on Information Theory, vol. 56, no. 3, pp. 1070-1084, Mar 2010.

[46] M. Costa, "Writing on dirty paper (corresp.)," IEEE Transactions on Information Theory, vol. 29, no. 3, pp. 439-441, May 1983.

[47] H. Weingarten, Y. Steinberg, and S. Shamai, "The capacity region of the gaussian multiple-input multiple-output broadcast channel," IEEE Transactions on Information Theory, vol. 52, no. 9, pp. 3936-3964, Sep 2006.

[48] M. Tomlinson, "New automatic equaliser employing modulo arithmetic," Electronics Letters, vol. 7, no. 5-6, p. $138,1971$.

[49] T. Yoo and A. Goldsmith, "Optimality of zero-forcing beamforming with multiuser diversity," in IEEE International Conference on Communications (ICC), 2005.

[50] — - "On the optimality of multiantenna broadcast scheduling using zero-forcing beamforming," IEEE Journal on Selected Areas in Communications, vol. 24, no. 3, pp. 528-541, Mar 2006.

[51] M. Joham, W. Utschick, and J. Nossek, "Linear transmit processing in MIMO communications systems," IEEE Transactions on Signal Processing, vol. 53, no. 8, pp. 2700-2712, Aug 2005.

[52] C. Peel, B. Hochwald, and A. Swindlehurst, "A vector-perturbation technique for near-capacity multiantenna multiuser communication_part i: Channel inversion and regularization," IEEE Transactions on Communications, vol. 53, no. 1, pp. 195-202, Jan 2005.

[53] W. Yang and G. Xu, "New method for designing smart antenna downlink weighting vectors based on the filter bank concept," in Advanced Signal Processing: Algorithms, Architectures, and Implementations VII, F. T. Luk, Ed. SPIE-Intl Soc Optical Eng, Oct 1997.

[54] V. K. Nguyen and J. S. Evans, "Multiuser transmit beamforming via regularized channel inversion: A large system analysis," in IEEE GLOBECOM 2008 - 2008 IEEE Global Telecommunications Conference, 2008.

[55] R. Muharar, R. Zakhour, and J. Evans, "Optimal power allocation and user loading for multiuser MISO channels with regularized channel inversion," IEEE Transactions on Communications, vol. 61, no. 12, pp. 5030-5041, Dec 2013.

[56] T. Lo, "Maximum ratio transmission," IEEE Transactions on Communications, vol. 47, no. 10, pp. 1458-1461, 1999.

[57] M. Bengtsson and B. Ottersten, "Optimal and suboptimal transmit beamforming," in Handbook of Antennas in Wireless Communications. CRC Press, 2001, pp. 18-1-18-33. 
[58] — _ "Optimal downlink beamforming using semidefinite optimization," in Proc. of 37th Annual Allerton Conference on Communication, Control, and Computing :, 1999, pp. 987-996.

[59] M. Schubert and H. Boche, "Solution of the multiuser downlink beamforming problem with individual SINR constraints," IEEE Transactions on Vehicular Technology, vol. 53, no. 1, pp. 18-28, Jan 2004.

[60] W. Yu and T. Lan, "Transmitter optimization for the multi-antenna downlink with per-antenna power constraints," IEEE Transactions on Signal Processing, vol. 55, no. 6, pp. 2646-2660, Jun 2007.

[61] H. Shen, W. Xu, A. L. Swindlehurst, and C. Zhao, "Transmitter optimization for per-antenna power constrained multiantenna downlinks: _newline an SLNR maximization methodology,” IEEE Transactions on Signal Processing, vol. 64, no. 10, pp. 2712-2725, May 2016.

[62] G. Dartmann, X. Gong, W. Afzal, and G. Ascheid, "On the duality of the max-min beamforming problem with per-antenna and per-antenna-array power constraints," IEEE Transactions on Vehicular Technology, vol. 62, no. 2, pp. 606-619, Feb 2013.

[63] G. Zheng, S. Chatzinotas, and B. Ottersten, "Generic optimization of linear precoding in multibeam satellite systems," IEEE Transactions on Wireless Communications, vol. 11, no. 6, pp. 2308-2320, Jun 2012.

[64] H. Dahrouj and W. Yu, "Coordinated beamforming for the multicell multi-antenna wireless system," IEEE Transactions on Wireless Communications, vol. 9, no. 5, pp. 1748-1759, May 2010.

[65] V.-D. Nguyen, L.-N. Tran, T. Duong, O.-S. Shin, and R. Farrell, "An efficient precoder design for multiuser MIMO cognitive radio networks with interference constraints," IEEE Transactions on Vehicular Technology, pp. 1-1, 2016.

[66] H. Zhang, C. Li, Y. Huang, and L. Yang, "Secure beamforming for SWIPT in multiuser MISO broadcast channel with confidential messages," IEEE Communications Letters, vol. 19, no. 8, pp. 1347-1350, Aug 2015.

[67] X. Chen and Y. Zhang, "Mode selection in MU-MIMO downlink networks: A physical-layer security perspective," IEEE Systems Journal, pp. 1-9, 2015.

[68] J. Xu, L. Liu, and R. Zhang, "Multiuser MISO beamforming for simultaneous wireless information and power transfer," IEEE Transactions on Signal Processing, vol. 62, no. 18, pp. 4798-4810, sep 2014.

[69] O. Mehanna, K. Huang, B. Gopalakrishnan, A. Konar, and N. D. Sidiropoulos, "Feasible point pursuit and successive approximation of non-convex QCQPs," IEEE Signal Processing Letters, vol. 22, no. 7, pp. 804-808, Jul 2015.

[70] O. T. Demir and T. E. Tuncer, "Antenna selection and hybrid beamforming for simultaneous wireless information and power transfer in multi-group multicasting systems," IEEE Transactions on Wireless Communications, vol. 15, no. 10, pp. 6948-6962, Oct 2016.

[71] X. Gao, L. Dai, S. Han, C.-L. I, and R. W. Heath, "Energy-efficient hybrid analog and digital precoding for MmWave MIMO systems with large antenna arrays," IEEE Journal on Selected Areas in Communications, vol. 34, no. 4, pp. 998-1009, Apr 2016.

[72] O. Mehanna, N. D. Sidiropoulos, and G. B. Giannakis, "Joint multicast beamforming and antenna selection," IEEE Transactions on Signal Processing, vol. 61, no. 10, pp. 2660-2674, May 2013.

[73] S. Ji, S. X. Wu, A. M.-C. So, and W.-K. Ma, "Multi-group multicast beamforming in cognitive radio networks via ranktwo transmit beamformed alamouti space-time coding," IEEE International Conference on Acoustic, Speech, and Signal Processing, 2013.

[74] zlem Tufe Demir and T. E. Tuncer, "Max-min fair resource allocation for swipt in multi-group multicast ofdm systems," to appear in IEEE Communications Letter, 2017.

[75] M. Sadeghi, L. Sanguinetti, and C. Yuen, "Hybrid precoding for multi-group physical layer multicasting," arXiv preprint arXiv:1703.10329, 2017. 
[76] B. Liu, L. Shi, and X.-G. Xia, "Robust rank-two multicast beamforming under a unified CSI uncertainty model," IEEE Signal Processing Letters, vol. 23, no. 10, pp. 1419-1423, Oct 2016.

[77] K. L. Law, X. Wen, M. T. Vu, and M. Pesavento, "General rank multiuser downlink beamforming with shaping constraints using real-valued ostbc," IEEE Transactions on Signal Processing, vol. 63, no. 21, pp. 5758-5771, 2015.

[78] A. Schad, K. L. Law, and M. Pesavento, "Rank-two beamforming and power allocation in multicasting relay networks." IEEE Trans. Signal Processing, vol. 63, no. 13, pp. 3435-3447, 2015.

[79] S. He, Y. Huang, S. Jin, and L. Yang, "Energy efficient coordinated beamforming design in multi-cell multicast networks," IEEE Communications Letters, vol. 19, no. 6, pp. 985-988, jun 2015.

[80] Y. Huang, Q. Li, W.-K. Ma, and S. Zhang, "Robust multicast beamforming for spectrum sharing-based cognitive radios," IEEE Transactions on Signal Processing, vol. 60, no. 1, pp. 527-533, Jan 2012.

[81] Y. Jeong, T. Q. S. Quek, J. S. Kwak, and H. Shin, "Multicasting in stochastic MIMO networks," IEEE Transactions on Wireless Communications, vol. 13, no. 4, pp. 1-13, Apr 2014.

[82] M. R. A. Khandaker and K.-K. Wong, "SWIPT in MISO multicasting systems," IEEE Wireless Communications Letters, vol. 3, no. 3, pp. 277-280, Jun 2014.

[83] D. W. K. Ng, R. Schober, and H. Alnuweiri, "Secure layered transmission in multicast systems with wireless information and power transfer," in IEEE International Conference on Communications (ICC). IEEE, 2014, pp. 5389-5395.

[84] J. Choi, "Iterative methods for physical-layer multicast beamforming," IEEE Transactions on Wireless Communications, vol. 14, no. 9, pp. 5185-5196, Sep 2015.

[85] S. X. Wu, W.-K. Ma, and A. M.-C. So, "Physical-layer multicasting by stochastic transmit beamforming and alamouti space-time coding," IEEE Transactions on Signal Processing, vol. 61, no. 17, pp. 4230-4245, Sep 2013.

[86] S. X. Wu, Q. Li, A. M.-C. So, and W.-K. Ma, "Rank-two beamforming and stochastic beamforming for miso physical-layer multicasting with finite-alphabet inputs," IEEE Signal Processing Letters, vol. 22, no. 10, pp. 1614 - 1618, 2015.

[87] S. Zhang, R. Zhang, and T. J. Lim, "MISO multicasting with constant envelope precoding," IEEE Wireless Communication letter, vol. 5, no. 6, pp. 588-591, December 2016.

[88] A. H. Phan, H. D. Tuan, H. H. Kha, and D. T. Ngo, "Nonsmooth optimization for efficient beamforming in cognitive radio multicast transmission," IEEE Transactions on Signal Processing, vol. 60, no. 6, pp. 2941-2951, 2012.

[89] M. Beko, "Efficient beamforming in cognitive radio multicast transmission," IEEE Transactions on Wireless Communications, vol. 11, no. 11, pp. 4108-4117, 2012.

[90] D. Taleb, Y. Liu, and M. Pesavento, "Full-rate general rank beamforming in single-group multicasting networks using non-orthogonal stbc," in Signal Processing Conference (EUSIPCO), 2016 24th European. IEEE, 2016, pp. $2365-2369$.

[91] Z. Xiang, M. Tao, and X. Wang, "Massive MIMO multicasting in noncooperative cellular networks," IEEE Journal on Selected Areas in Communications, vol. 32, no. 6, pp. 1180-1193, Jun 2014.

[92] M. Sadek, A. Tarighat, and A. Sayed, "A leakage-based precoding scheme for downlink multi-user MIMO channels," IEEE Transactions on Wireless Communications, vol. 6, no. 5, pp. 1711-1721, May 2007.

[93] L.-U. Choi and R. Murch, "A transmit preprocessing technique for multiuser MIMO systems using a decomposition approach,” IEEE Transactions on Wireless Communications, vol. 3, no. 1, pp. 20-24, Jan 2004.

[94] D. Spano, M. Alodeh, S. Chatzinotas, and B. Ottersten, "Symbol-level precoding for the non-linear multiuser MISO downlink channel," submitted to IEEE Transactions on Signal Processing, 2016.

[95] A. Wiesel, Y. Eldar, and S. Shamai, “Zero-forcing precoding and generalized inverses," IEEE Transactions on Signal Processing, vol. 56, no. 9, pp. 4409-4418, Sep 2008.

[96] K.-K. Wong, R. Murch, and K. Letaief, “A joint-channel diagonalization for multiuser MIMO antenna systems,” IEEE Transactions on Wireless Communications, vol. 24, no. 5, pp. 773-786, May 2003. 
[97] C. Masouros and E. Alsusa, "Dynamic linear precoding for the exploitation of known interference in MIMO broadcast systems," IEEE Transactions on Wireless Communications, vol. 8, no. 3, pp. 1396-1404, Mar 2009.

[98] C. Masouros, "Correlation rotation linear precoding for MIMO broadcast communications," IEEE Transactions on Signal Processing, vol. 59, no. 1, pp. 252-262, Jan 2011.

[99] F. A. Khan, C. Masouros, and T. Ratnarajah, "Interference-driven linear precoding in multiuser MISO downlink cognitive radio network," IEEE Transactions on Vehicular Technology, vol. 61, no. 6, pp. 2531-2543, Jul 2012.

[100] C. Masouros and T. Ratnarajah, "Interference as a source of green signal power in cognitive relay assisted co-existing MIMO wireless transmissions," IEEE Transactions on Communications, vol. 60, no. 2, pp. 525-536, Feb 2012.

[101] C. Masouros, M. Sellathurai, and T. Ratnarajah, "Interference optimization for transmit power reduction in tomlinsonharashima precoded MIMO downlinks," IEEE Transactions on Signal Processing, vol. 60, no. 5, pp. 2470-2481, May 2012.

[102] M. Alodeh, D. Spano, S. Chatzinotas, and B. Ottersten, "Peak power minimization in symbol-level precoding for cognitive MISO downlink channels," in IEEE Digital Signal Processing conference (DSP), Oct 2016.

[103] M. Alodeh, S. Chatzinotas, and B. Ottersten, "Symbol-level multiuser miso precoding for multi-level adaptive modulation," submitted to IEEE Transactions on Wireless Communications.

[104] O. Tervo, L.-N. Tran, and M. Juntti, "Optimal energy-efficient transmit beamforming for multi-user MISO downlink," IEEE Transactions on Signal Processing, vol. 63, no. 20, pp. 5574-5588, Oct 2015.

[105] C. Masouros, M. Sellathurai, and T. Ratnarajah, "Maximizing energy efficiency in the vector precoded MU-MISO downlink by selective perturbation," IEEE Transactions on Wireless Communications, vol. 13, no. 9, pp. 4974-4984, Sep 2014.

[106] O. Tervo, L.-N. Tran, H. Pennanen, S. Chatzinotas, M. Juntti, and B. Ottersten, "Energy-efficient coordinated multi-cell multigroup multicast beamforming with antenna selection," arXiv preprint arXiv:1702.05632, 2017.

[107] S. Shi, M. Schubert, and H. Boche, "Rate optimization for multiuser mimo systems with linear processing," IEEE Transactions on Signal Processing, vol. 56, no. 8, pp. 4020-4030, 2008.

[108] H. Boche and M. Schubert, "Resource allocation in multiantenna systems-achieving max-min fairness by optimizing a sum of inverse SIR," IEEE Transactions on Signal Processing, vol. 54, no. 6, pp. 1990-1997, Jun 2006.

[109] Y. Wu, C. Xiao, X. Gao, J. D. Matyjas, and Z. Ding, "Linear precoder design for MIMO interference channels with finite-alphabet signaling," IEEE Transactions on Communications, vol. 61, no. 9, pp. 3766-3780, Sep 2013.

[110] R. Ghaffar and R. Knopp, "Near optimal linear precoder for multiuser MIMO for discrete alphabets," in 2010 IEEE International Conference on Communications, May 2010.

[111] M. Shenouda and T. Davidson, "Nonlinear and linear broadcasting with QoS requirements: Tractable approaches for bounded channel uncertainties," IEEE Transactions on Signal Processing, vol. 57, no. 5, pp. 1936-1947, May 2009.

[112] D. Ponukumati, F. Gao, and M. Bode, "Robust multicell downlink beamforming based on second-order statistics of channel state information," in 2011 IEEE Global Telecommunications Conference - GLOBECOM 2011, Dec 2011.

[113] N. Vucic and H. Boche, "Robust QoS-constrained optimization of downlink multiuser MISO systems," IEEE Transactions on Signal Processing, vol. 57, no. 2, pp. 714-725, Feb 2009.

[114] C. Zhang, W. Xu, and M. Chen, "Robust MMSE beamforming for multiuser MISO systems with limited feedback," IEEE Signal Processing Letters, vol. 16, no. 7, pp. 588-591, Jul 2009.

[115] J. Wang and D. Palomar, "Worst-case robust MIMO transmission with imperfect channel knowledge," IEEE Transactions on Signal Processing, vol. 57, no. 8, pp. 3086-3100, Aug 2009.

[116] W.-K. Ma, J. Pan, A. M.-C. So, and T.-H. Chang, "Unraveling the rank-one solution mystery of robust MISO downlink 
transmit optimization: A verifiable sufficient condition via a new duality result," IEEE Transactions on Signal Processing, vol. 65, no. 7, pp. 1909-1924, Apr 2017.

[117] G. Zheng, S. Ma, K.-K. Wong, and T.-S. Ng, "Robust beamforming in the MISO downlink with quadratic channel estimation and optimal training," IEEE Transactions on Wireless communications, vol. 8, no. 3, pp. 1067-1072, 2009.

[118] K.-Y. Wang, A. M.-C. So, T.-H. Chang, W.-K. Ma, and C.-Y. Chi, "Outage constrained robust transmit optimization for multiuser MISO downlinks: Tractable approximations by conic optimization," IEEE Transactions on Signal Processing, vol. 62, no. 21, pp. 5690-5705, Nov 2014.

[119] H. Joudeh and B. Clerckx, "Robust transmission in downlink multiuser MISO systems: A rate-splitting approach,” IEEE Transactions on Signal Processing, vol. 64, no. 23, pp. 6227-6242, Dec 2016.

[120] F. Sohrabi and T. N. Davidson, "Coordinate update algorithms for robust power loading for the MU-MISO downlink with outage constraints," IEEE Transactions on Signal Processing, vol. 64, no. 11, pp. 2761-2773, Jun 2016.

[121] M. Medra, Y. Huang, W.-K. Ma, and T. N. Davidson, "Low-complexity robust MISO downlink precoder design under imperfect CSI," IEEE Transactions on Signal Processing, vol. 64, no. 12, pp. 3237-3249, Jun 2016.

[122] D. Kwon, H. S. Kang, and D. K. Kim, "Robust interference exploitation-based precoding scheme with quantized CSIT," IEEE Communications Letters, vol. 20, no. 4, pp. 780-783, Apr 2016.

[123] D. Kwon, W.-Y. Yeo, and D. K. Kim, "A new precoding scheme for constructive superposition of interfering signals in multiuser MIMO systems," IEEE Communications Letters, vol. 18, no. 11, pp. 2047-2050, Nov 2014.

[124] C. Masouros and G. Zheng, "Exploiting known interference as green signal power for downlink beamforming optimization," IEEE Transactions on Signal Processing, vol. 63, no. 14, pp. 3628-3640, Jul 2015.

[125] S. K. Mohammed and E. G. Larsson, "Per-antenna constant envelope precoding for large multi-user MIMO systems," IEEE Transactions on Communications, vol. 61, no. 3, pp. 1059-1071, Mar 2013.

[126] _ _Constant-envelope multi-user precoding for frequency-selective massive MIMO systems," IEEE Wireless Communications Letters, vol. 2, no. 5, pp. 547-550, Oct 2013.

[127] P. V. Amadori and C. Masouros, "Constant envelope precoding by interference exploitation in phase shift keying-modulated multiuser transmission," IEEE Transactions on Wireless Communications, vol. 16, no. 1, pp. 538-550, Jan 2017.

[128] W.-C. Liao, T.-H. Chang, W.-K. Ma, and C.-Y. Chi, "QoS-based transmit beamforming in the presence of eavesdroppers: An optimized artificial-noise-aided approach,” IEEE Transactions on Signal Processing, vol. 59, no. 3, pp. 1202-1216, Mar 2011.

[129] W. Wu, S. Wu, and B. Wang, "Robust multi-objective beamforming design for power efficient and secure communication in mu-miso networks," IEEE Access, 2017.

[130] K.-J. Lee and I. Lee, "MMSE based block diagonalization for cognitive radio mimo broadcast channels," IEEE Transactions in Wireless Cmmunications, vol. 10, no. 10, pp. 3139 - 3144, Oct 2011.

[131] X. Zhang, A. Molisch, and S.-Y. Kung, "Variable-phase-shift-based RF-baseband codesign for MIMO antenna selection," IEEE Transactions on Signal Processing, vol. 53, no. 11, pp. 4091-4103, Nov 2005.

[132] O. E. Ayach, S. Rajagopal, S. Abu-Surra, Z. Pi, and R. W. Heath, "Spatially sparse precoding in millimeter wave MIMO systems," IEEE Transactions on Wireless Communications, vol. 13, no. 3, pp. 1499-1513, Mar 2014.

[133] C. G. Tsinos, S. Maleki, S. Chatzinotas, and B. Ottersten, "On the energy-efficiency of hybrid analog-digital transceivers for single-and multi-carrier large antenna array systems," IEEE Journal on Selected Areas in Communications, vol. 35, no. 9, pp. 1980-1995, 2017.

[134] — - "Hybrid analog-digital transceiver designs for cognitive large-scale antenna array systems," submitted to IEEE Trans. on Signal Processing, 2017. 
[135] A. Alkhateeb and R. W. Heath, "Frequency selective hybrid precoding for limited feedback millimeter wave systems," IEEE Transactions on Communications, vol. 64, no. 5, pp. 1801-1818, May 2016.

[136] D. Christopoulos, S. Chatzinotas, G. Zheng, J. Grotz, and B. Ottersten, "Linear and nonlinear techniques for multibeam joint processing in satellite communications," EURASIP journal on wireless communications and networking, vol. 2012, no. 1, p. 162, 2012.

[137] S. Jacobsson, G. Durisi, M. Coldrey, T. Goldstein, and C. Studer, "Quantized precoding for massive mu-mimo," to appear in IEEE Transactions on Communications, 2017.

[138] H. Jedda, J. A. Nossek, and A. Mezghani, "Minimum ber precoding in 1-bit massive MIMO systems," in Sensor Array and Multichannel Signal Processing Workshop (SAM), 2016 IEEE. IEEE, 2016, pp. 1-5.

[139] M. Kazemi, H. Aghaeinia, and T. M. Duman, "Discrete-phase constant envelope precoding for massive mimo systems," IEEE Transactions on Communications, vol. 65, no. 5, pp. 2011 - 2021, May 2017.

[140] C. Risi, D. Persson, and E. G. Larsson, "Massive MIMO with 1-bit ADC," available on Arxiv [cs.IT]: 1404.7736v1.

[141] N. Bornhorst and M. Pesavento, "An iterative convex approximation approach for transmit beamforming in multi-group multicasting," in Signal Processing Advances in Wireless Communications (SPAWC), 2011 IEEE 12th International Workshop on. IEEE, 2011, pp. 426-430.

[142] T.-H. Chang, Z.-Q. Luo, and C.-Y. Chi, "Approximation bounds for semidefinite relaxation of max-min-fair multicast transmit beamforming problem," IEEE Transactions on Signal Processing, vol. 56, no. 8, pp. 3932-3943, 2008.

[143] M. Sadeghi, E. Björnson, E. G. Larsson, C. Yuen, and T. L. Marzetta, "Max-min fair transmit precoding for multi-group multicasting in massive mimo," submitted, 2017.

[144] Arapoglou, A. Ginesi, T. G, D. Christopoulos, S. Chatzinotas, B. Ottersten, M. Ángel Vázquez, A. Pèrez-Neira, S. Andrenacci, and A. Vanelli-Coralli, "Joint transmitter signal processing in multi-beam satellite systems," Patent PCT/EP2015/058 023, 2015.

[145] O. Tervo, L.-N. Tran, H. Pennanen, and a. M. J. a. O. Symeon Chatzinotas, Energy-Efficient Coordinated Multi-Cell Multigroup Multicast Beamforming with Antenna Selection, May 2017.

[146] N. Bornhorst and M. Pesavento, "Beamforming for multi-group multicasting with statistical channel state information using second-order cone programming," in Acoustics, Speech and Signal Processing (ICASSP), 2012 IEEE International Conference on. IEEE, 2012, pp. 3237-3240.

[147] Z. Xiang, M. Tao, and X. Wang, "Coordinated multicast beamforming in multicell networks," IEEE Transactions on Wireless Communications, vol. 12, no. 1, pp. 12-21, jan 2013.

[148] N. Bornhorst, M. Pesavento, and A. B. Gershman, "Distributed beamforming for multi-group multicasting relay networks," IEEE Transactions on Signal Processing, vol. 60, no. 1, pp. 221-232, jan 2012.

[149] E. Chen and M. Tao, "Admm-based fast algorithm for multi-group multicast beamforming in large-scale wireless systems," IEEE Transactions on Communication, vol. 65, no. 6, pp. 2685 - 2698, jUNE 2017.

[150] G.-W. Hsu, B. Liu, H.-H. Wang, and H.-J. Su, "Joint beamforming for multicell multigroup multicast with per-cell power constraints," IEEE Transactions on Vehicular Technology, vol. 66, no. 5, pp. 4044-4058, 2017.

[151] Y.-W. P. Hong, W.-C. Li, T.-H. Chang, and C.-H. Lee, "Coordinated multicasting with opportunistic user selection in multicell wireless systems.” IEEE Trans. Signal Processing, vol. 63, no. 13, pp. 3506-3521, 2015.

[152] B. Clerckx, H. Joudeh, C. Hao, M. Dai, and B. Rassouli, "Rate splitting for MIMO wireless networks: a promising PHY-layer strategy for LTE evolution,” IEEE Communications Magazine, vol. 54, no. 5, pp. 98-105, May 2016.

[153] H. Joudeh and B. Clerckx, "Sum-rate maximization for linearly precoded downlink multiuser miso systems with partial csit: A rate-splitting approach," IEEE Transactions on Communications, vol. 64, no. 11, pp. 4847 - 4861, November 2016. 
[154] D. Christopoulos, S. Chatzinotas, and B. Ottersten, "Cellular-broadcast service convergence through caching for CoMP cloud RANs," in 2015 IEEE Symposium on Communications and Vehicular Technology in the Benelux (SCVT), Nov 2015.

[155] E. G. Larsson and H. V. Poor, "Joint beamforming and broadcasting in massive MIMO," IEEE Transactions on Wireless Communications, vol. 15, no. 4, pp. 3058-3070, Apr 2016.

[156] M. Tao, E. Chen, H. Zhou, and W. Yu, "Content-centric sparse multicast beamforming for cache-enabled cloud RAN," IEEE Transactions on Wireless Communications, vol. 15, no. 9, pp. 6118-6131, Sep 2016.

[157] B.-X. Wu, K. C.-J. Lin, K.-C. Hsu, and H.-Y. Wei, "HybridCast: Joint multicast-unicast design for multiuser MIMO networks," in 2015 IEEE Conference on Computer Communications (INFOCOM), Apr 2015.

[158] J. Zhao, O. Simeone, D. G. üz, and D. Gómez-Barquero, "Non-orthogonal unicast and broadcast transmission via joint beamforming and ldm in cellular networks," IEEE Global Communications Conference (GLOBECOM), 2016.

[159] J. Zander and P. Mhnen, "Riding the data tsunami in the cloud: myths and challenges in future wireless access," IEEE Communications Magazine, vol. 51, no. 3, pp. 145-151, Mar 2013.

[160] Q. Spencer, A. Swindlehurst, and M. Haardt, "Zero-forcing methods for downlink spatial multiplexing in multiuser MIMO channels," IEEE Transactions on Signal Processing, vol. 52, no. 2, pp. 461-471, Feb 2004.

[161] A. Kalantari, M. Soltanalian, S. Maleki, S. Chatzinotas, and B. Ottersten, "Directional modulation via symbol-level precoding: A way to enhance security," IEEE Journal of Selected Topics in Signal Processing, vol. 10, no. 8, pp. 14781493, Dec 2016.

[162] A. Kalantari, C. Tsinos, M. Soltanalian, S. Chatzinotas, W.-K. Ma, and B. Ottersten, "Energy-efficient mqam precoder design with spatial peak power minimization for MIMO directional modulation transceivers," online:https://arxiv.org/pdf/1702.06878.pdf, 2017.

[163] E. Baghdady, "Directional signal modulation by means of switched spaced antennas," IEEE Transactions on Communications, vol. 38, no. 4, pp. 399-403, Apr 1990.

[164] M. P. Daly and J. T. Bernhard, "Beamsteering in pattern reconfigurable arrays using directional modulation," IEEE Transactions on Antennas and Propagation, vol. 58, no. 7, pp. 2259-2265, Jul 2010.

[165] Q. Zhu, S. Yang, R. Yao, and Z. Nie, "Directional modulation based on 4-d antenna arrays," IEEE Transactions on Antennas and Propagation, vol. 62, no. 2, pp. 621-628, Feb 2014.

[166] A. Babakhani, D. B. Rutledge, and A. Hajimiri, "Transmitter architectures based on near-field direct antenna modulation," IEEE Journal of Solid-State Circuits, vol. 43, no. 12, pp. 2674-2692, Dec 2008.

[167] A. H. Chang, A. Babakhani, and A. Hajimiri, "Near-field direct antenna modulation (NFDAM) transmitter at 2.4GHz," in 2009 IEEE Antennas and Propagation Society International Symposium, Jun 2009.

[168] J. Lavaei, A. Babakhani, A. Hajimiri, and J. C. Doyle, "A study of near-field direct antenna modulation systems using convex optimization," in Proceedings of the 2010 American Control Conference, Jun 2010.

[169] M. Daly and J. Bernhard, "Directional modulation technique for phased arrays," IEEE Transactions on Antennas and Propagation, vol. 57, no. 9, pp. 2633-2640, Sep 2009.

[170] M. P. Daly, E. L. Daly, and J. T. Bernhard, "Demonstration of directional modulation using a phased array," IEEE Transactions on Antennas and Propagation, vol. 58, no. 5, pp. 1545-1550, May 2010.

[171] M. P. Daly and J. T. Bernhard, "Directional modulation and coding in arrays," in 2011 IEEE International Symposium on Antennas and Propagation (APSURSI), Jul 2011.

[172] B. Guo, Y. hong Yang, G. Xin, and Y. qun Tang, "Combinatorial interference directional modulation for physical layer security transmission," in IEEE Information Technology, Networking, Electronic and Automation Control Conference, May 2016. 
[173] T. Hong, M.-Z. Song, and Y. Liu, "Dual-beam directional modulation technique for physical-layer secure communication," IEEE Antennas and Wireless Propagation Letters, vol. 10, pp. 1417-1420, 2011.

[174] N. Valliappan, A. Lozano, and R. W. Heath, "Antenna subset modulation for secure millimeter-wave wireless communication," IEEE Transactions on Communications, vol. 61, no. 8, pp. 3231-3245, Aug 2013.

[175] Y. Ding and V. Fusco, "BER-driven synthesis for directional modulation secured wireless communication," International Journal of Microwave and Wireless Technologies, vol. 6, no. 02, pp. 139-149, Nov 2013.

[176] _ - "Directional modulation transmitter radiation pattern considerations," IET Microwaves, Antennas \& Propagation, vol. 7, no. 15, pp. 1201-1206, Dec 2013.

[177] Y. Ding and V. F. Fusco, "Constraining directional modulation transmitter radiation patterns," IET Microwaves, Antennas \& Propagation, vol. 8, no. 15, pp. 1408-1415, Dec 2014.

[178] H. Shi and A. Tennant, "Enhancing the security of communication via directly modulated antenna arrays," IET Microwaves, Antennas \& Propagation, vol. 7, no. 8, pp. 606-611, Jun 2013.

[179] Y. Ding and V. F. Fusco, "Establishing metrics for assessing the performance of directional modulation systems," IEEE Transactions on Antennas and Propagation, vol. 62, no. 5, pp. 2745-2755, May 2014.

[180] J. Hu, F. Shu, and J. Li, "Robust synthesis method for secure directional modulation with imperfect direction angle," IEEE Communications Letters, vol. 20, no. 6, pp. 1084-1087, Jun 2016.

[181] Y. Ding and V. F. Fusco, "Directional modulation far-field pattern separation synthesis approach," IET Microwaves, Antennas \& Propagation, vol. 9, no. 1, pp. 41-48, Jan 2015.

[182] Y. Ding and V. Fusco, "Directional modulation-enhanced retrodirective array," Electronics Letters, vol. 51, no. 1, pp. 118-120, Jan 2015.

[183] Y. Ding and V. F. Fusco, "MIMO-inspired synthesis of directional modulation systems," IEEE Antennas and Wireless Propagation Letters, vol. 15, pp. 580-584, 2016.

[184] — - "A vector approach for the analysis and synthesis of directional modulation transmitters," IEEE Transactions on Antennas and Propagation, vol. 62, no. 1, pp. 361-370, Jan 2014.

[185] Y. Ding and V. Fusco, "Orthogonal vector approach for synthesis of multi-beam directional modulation transmitters," IEEE Antennas and Wireless Propagation Letters, vol. 14, pp. 1330-1333, 2015.

[186] H. Shi and A. Tennant, "Simultaneous, multichannel, spatially directive data transmission using direct antenna modulation," IEEE Transactions on Antennas and Propagation, vol. 62, no. 1, pp. 403-410, Jan 2014.

[187] M. Hafez and H. Arslan, "On directional modulation: An analysis of transmission scheme with multiple directions," in 2015 IEEE International Conference on Communication Workshop (ICCW), Jun 2015.

[188] M. Hafez, T. Khattab, T. Elfouly, and H. Arslan, "Secure multiple-users transmission using multi-path directional modulation," in 2016 IEEE International Conference on Communications (ICC), May 2016.

[189] Y. Ding and V. Fusco, "A synthesis-free directional modulation transmitter using retrodirective array," IEEE Journal of Selected Topics in Signal Processing, vol. 11, no. 2, pp. 428-441, Mar 2016.

[190] A. Kalantari, M. Soltanalian, S. Maleki, S. Chatzinotas, and B. Ottersten, "Secure M-PSK communication via directional modulation," in 2016 IEEE International Conference on Acoustics, Speech and Signal Processing (ICASSP), Mar 2016.

[191] P. Cheng, M. Tao, and W. Zhang, “A new slnr-based linear precoding for downlink multi-user multi-stream mimo systems," IEEE Communications Letters, vol. 14, no. 11, pp. 1008-1010, 2010.

[192] P. Patcharamaneepakorn, S. Armour, and A. Doufexi, "On the equivalence between slnr and mmse precoding schemes with single-antenna receivers," IEEE Communications Letters, vol. 16, no. 7, pp. 1034-1037, 2012.

[193] H. Joudeh and B. Clerckx, "A rate-splitting strategy for max-min fair multigroup multicasting," in 2016 IEEE 17th International Workshop on Signal Processing Advances in Wireless Communications (SPAWC), Jul 2016. 
[194] S. S. Christensen, R. Agarwal, E. D. Carvalho, and J. M. Cioffi, "Weighted sum-rate maximization using weighted mmse for mimo-bc beamforming design," IEEE Transactions on Wireless Communications, vol. 7, no. 12, pp. 4792 - 4799 , December 2008 .

[195] N. Ravindran and N. Jindal, "Limited feedback-based block diagonalization for the MIMO broadcast channel," IEEE Journal on Selected Areas in Communications, vol. 26, no. 8, pp. 1473-1482, Oct 2008.

[196] S. A. A. Fakoorian and A. L. Swindlehurst, "On the optimality of linear precoding for secrecy in the MIMO broadcast channel," IEEE Journal on Selected Areas in Communications, vol. 31, no. 9, pp. 1701-1713, Sep 2013.

[197] A. Kalantari, G. Zheng, Z. Gao, Z. Han, and B. Ottersten, "Secrecy analysis on network coding in bidirectional multibeam satellite communications," IEEE Transactions on Information Forensics and Security, vol. 10, no. 9, pp. 1862-1874, Sep 2015.

[198] K. An, M. Lin, J. Ouyang, and W.-P. Zhu, "Secure transmission in cognitive satellite terrestrial networks," IEEE Journal on Selected Areas in Communications, vol. 34, no. 11, pp. 3025-3037, Nov 2016.

[199] C. Song, J. Park, B. Clerckx, I. Lee, and K.-J. Lee, "Generalized precoder designs based on weighted mmse criterion for energy harvesting constrained mimo and multi-user mimo channels," IEEE Transactions on Wireless Communications, vol. 15, no. 12, pp. 7941-7954, 2016.

[200] S. Timotheou, I. Krikidis, G. Zheng, and B. Ottersten, "Beamforming for miso interference channels with qos and rf energy transfer," IEEE Transactions on Wireless Communications, vol. 13, no. 5, pp. 2646-2658, 2014.

[201] Q. Li, Q. Zhang, and J. Qin, "A special class of fractional qcqp and its applications on cognitive collaborative beamforming.” IEEE Trans. Signal Processing, vol. 62, no. 8, pp. 2151-2164, 2014.

[202] A. H. Phan, H. D. Tuan, H. H. Kha, and D. T. Ngo, "Nonsmooth optimization for beamforming in cognitive multicast transmission," in Global Telecommunications Conference (GLOBECOM 2010), 2010 IEEE. IEEE, 2010, pp. 1-5.

[203] O. Tervo, L.-N. Tran, S. Chatzinotas, M. J. Juntti, and B. E. Ottersten, "Energy-efficient joint unicast and multicast beamforming with multi-antenna user terminal," IEEE International Workshop on Signal Processing Advances in Wireless Communications (SPAWC 2017), 2017.

[204] D. Lee, H. Seo, B. Clerckx, E. Hardouin, D. Mazzarese, S. Nagata, and K. Sayana, "Coordinated multipoint transmission and reception in LTE-advanced: deployment scenarios and operational challenges," IEEE Communications Magazine, vol. 50, no. 2, pp. 148-155, Feb 2012.

[205] E. G. Larsson, "Joint beamforming and broadcasting in massive MIMO," in 2015 IEEE 16th International Workshop on Signal Processing Advances in Wireless Communications (SPAWC), Jun 2015.

[206] M. A. Sedaghat, A. Bereyhi, and R. R. Müller, "A new class of nonlinear precoders for hardware efficient massive MIMO systems," arXiv preprint arXiv:1704.08469, 2017.

[207] C. G. Tsinos, S. Maleki, S. Chatzinotas, and B. Ottersten, "Hybrid analog-digital transceiver designs for cognitive millimeter wave radio systems," in Asilomar Conference on Signals, Systems, and Computers, Nov 2016.

[208] T. E. Bogale, L. B. Le, A. Haghighat, and L. Vandendorpe, "On the number of rf chains and phase shifters, and scheduling design with hybrid analog-digital beamforming," IEEE Transactions on Wireless Communications, vol. 15, no. 5, pp. 3311-3326, 2016.

[209] M. Alodeh, S. Chatzinotas, and B. Ottersten, "A multicast approach for constructive interference precoding in MISO downlink channel," in 2014 IEEE International Symposium on Information Theory, Jun 2014.

[210] — "Constructive multiuser interference in symbol level precoding for the MISO downlink channel," IEEE Transactions on Signal Processing, vol. 63, no. 9, pp. 2239-2252, may 2015.

[211] _ _ "Energy efficient symbol-level precoding in multiuser MISO channels," in 2015 IEEE 16th International Workshop on Signal Processing Advances in Wireless Communications (SPAWC), Jun 2015. 
[212] - "Symbol based precoding in the downlink of cognitive MISO channel," in Lecture Notes of the Institute for Computer Sciences, Social Informatics and Telecommunications Engineering. Springer Nature, 2015, pp. 370-380.

[213] — , "Constructive interference through symbol level precoding for multi-level modulation," in 2015 IEEE Global Communications Conference (GLOBECOM), Dec 2015.

[214] — , "Energy-efficient symbol-level precoding in multiuser MISO based on relaxed detection region," IEEE Transactions on Wireless Communications, vol. 15, no. 5, pp. 3755-3767, May 2016.

[215] B. O. Maha Alodeh, Symeon Chatzinotas, "Method and device for symbol-level multiuser precoding," Patent PCT/EP2016/072 600, 2016.

[216] D. Spano, M. Alodeh, S. Chatzinotas, and B. Ottersten, "Per-antenna power minimization in symbol-level precoding," in 2016 IEEE Global Communications Conference (GLOBECOM), Dec 2016.

[217] K. Ntougias, D. Ntaikos, and C. B. Papadias, "Robust low-complexity arbitrary user- and symbol-level multi-cell precoding with single-fed load-controlled parasitic antenna arrays," in International Conference on Telecommunications (ICT), May 2016.

[218] J. Zhang, Y. Huang, J. Wang, B. Ottersten, and L. Yang, "Per-antenna constant envelope precoding and antenna subset selection: A geometric approach,” IEEE Transactions on Signal Processing, vol. 64, no. 23, pp. 6089-6104, 2016.

[219] J. Xu, L. Duan, and R. Zhang, “Transmit optimization for symbol-level spoofing,” [cs.IT]:1608.00722v1.

[220] M. R. A. Khandaker, C. Masouros, and K.-K. Wong, "Constructive interference based secure precoding: A new dimension in physical layer security," submitted to IEEE Transactions on Signal Processing, 2016.

[221] S. Timotheou, G. Zheng, C. Masouros, and I. Krikidis, "Exploiting constructive interference for simultaneous wireless information and power transfer in multiuser downlink systems," IEEE Journal on Selected Areas in Communications, vol. 34, no. 5, pp. 1772-1784, May 2016.

[222] S. K. Mohammed and E. G. Larsson, "Single-user beamforming in large-scale MISO systems with per-antenna constantenvelope constraints: The doughnut channel," IEEE Transactions on Wireless Communications, vol. 11, no. 11, pp. 3992-4005, Nov 2012.

[223] J. Pan and W.-K. Ma, "Constant envelope precoding for single-user large-scale MISO channels: Efficient precoding and optimal designs," IEEE Journal of Selected Topics in Signal Processing, vol. 8, no. 5, pp. 982-995, Oct 2014.

[224] J.-C. Chen, C.-K. Wen, and K.-K. Wong, "Improved constant envelope multiuser precoding for massive MIMO systems," IEEE Communications Letters, vol. 18, no. 8, pp. 1311-1314, Aug 2014.

[225] S. Mukherjee and S. K. Mohammed, "Constant-envelope precoding with time-variation constraint on the transmitted phase angles," IEEE Wireless Communications Letters, vol. 4, no. 2, pp. 221-224, Apr 2015.

[226] J. Zhang, Y. Huang, J. Wang, B. Ottersten, and L. Yang, "Per-antenna constant envelope precoding and antenna subset selection: A geometric approach," IEEE Transactions on Signal Processing, vol. 64, no. 23, pp. 6089-6104, Dec 2016.

[227] S. Zhang, R. Zhang, and T. J. Lim, "Receive beamforming optimization for MIMO system with constant envelope precoding," in 2016 IEEE International Conference on Communications (ICC), May 2016.

[228] — - "Constant envelope precoding with adaptive receiver constellation in MISO fading channel," IEEE Transactions on Wireless Communications, vol. 15, no. 10, pp. 6871-6882, Oct 2016.

[229] S. Boyd and L. Vandenberghe, Convex Optimization. Cambridge University Press, 2004.

[230] S. M. Razavi and T. Ratnarajah, "Adaptively regularized phase alignment precoding for multiuser multiantenna downlink," IEEE Transactions on Vehicular Technology, vol. 64, no. 10, pp. 4863-4869, Oct 2015.

[231] A. Gershman, N. Sidiropoulos, S. Shahbazpanahi, M. Bengtsson, and B. Ottersten, "Convex optimization-based beamforming," IEEE Signal Processing Magazine, vol. 27, no. 3, pp. 62-75, May 2010. 
[232] N. Jindal, “Antenna combining for the MIMO downlink channel,” IEEE Transactions on Wireless Communications, vol. 7, no. 10 , pp. 3834-3844, Oct 2008.

[233] M. Trivellato, F. Boccardi, and H. Huang, "On transceiver design and channel quantization for downlink multiuser MIMO systems with limited feedback," IEEE Journal on Selected Areas in Communications, vol. 26, no. 8, pp. 1494-1504, Oct 2008.

[234] R. Chen, Z. Shen, J. G. Andrews, and R. W. Heath, "Multimode transmission for multiuser MIMO systems with block diagonalization," IEEE Transactions on Signal Processing, vol. 56, no. 7, pp. 3294-3302, Jul 2008.

[235] C. Guthy, W. Utschick, R. Hunger, and M. Joham, "Efficient weighted sum rate maximization with linear precoding," IEEE Transactions on Signal Processing, vol. 58, no. 4, pp. 2284-2297, Apr 2010.

[236] A. Bayesteh and A. K. Khandani, "On the user selection for MIMO broadcast channels," IEEE Transactions on Information Theory, vol. 54, no. 3, pp. 1086-1107, Mar 2008.

[237] L. Zheng and D. Tse, "Diversity and multiplexing: a fundamental tradeoff in multiple-antenna channels," IEEE Transactions on Information Theory, vol. 49, no. 5, pp. 1073-1096, May 2003.

[238] A. Lozano and N. Jindal, "Transmit diversity vs. spatial multiplexing in modern MIMO systems," IEEE Transactions on Wireless Communications, vol. 9, no. 1, pp. 186-197, Jan 2010.

[239] E. Björnson, M. Kountouris, M. Bengtsson, and B. Ottersten, "Receive combining vs. multi-stream multiplexing in downlink systems with multi-antenna users," IEEE Transactions on Signal Processing, vol. 61, no. 13, pp. 3431-3446, Jul 2013.

[240] M. Dohler, R. W. Heath, A. Lozano, C. B. Papadias, and R. A. Valenzuela, "Is the PHY layer dead?" IEEE Communications Magazine, vol. 49, no. 4, pp. 159-165, Apr 2011.

[241] E. Matskani, N. Sidiropoulos, Z.-Q. Luo, and L. Tassiulas, "Efficient batch and adaptive approximation algorithms for joint multicast beamforming and admission control," IEEE Transactions on Signal Processing, vol. 57, no. 12, pp. 4882-4894, Dec 2009.

[242] M. Alodeh, S. Chatzinotas, and B. Ottersten, "User selection for symbol-level multigroup multicasting precoding in the downlink of miso channels," in to be submitted., 2017.

[243] E. Bjrnson, E. G. Larsson, and T. L. Marzetta, "Massive MIMO: ten myths and one critical question," IEEE Communications Magazine, vol. 54, no. 2, pp. 114-123, Feb 2016.

[244] Y. Li, C. Tao, G. Seco-Granados, A. Mezghani, A. L. Swindlehurst, and L. Liu, "Channel estimation and performance analysis of one-bit massive mimo systems," Submitted, available on arXiv [cs.IT]:1609.07427v2.

[245] F. Rusek, D. Persson, B. K. Lau, E. G. Larsson, T. L. Marzetta, and F. Tufvesson, "Scaling up MIMO: Opportunities and challenges with very large arrays," IEEE Signal Processing Magazine, vol. 30, no. 1, pp. 40-60, Jan 2013.

[246] A. Gokceoglu, E. Björnson, E. G. Larsson, and M. Valkama, "Spatio-temporal waveform design for multi-user massive MIMO downlink with 1-bit receivers," IEEE Journal of Selected Topics in Signal Processing, pp. 1-1, 2016.

[247] E. Björnson, M. Matthaiou, and M. Debbah, "Massive MIMO with non-ideal arbitrary arrays: Hardware scaling laws and circuit-aware design," IEEE Transactions on Wireless Communications, vol. 14, no. 8, pp. 4353-4368, Aug 2015.

[248] S. Jacobsson, G. Durisi, M. Coldrey, T. Goldstein, and C. Studer, "Nonlinear 1-bit precoding for massive MU-MIMO with higher-order modulation," in Signals, Systems and Computers, 2016 50th Asilomar Conference on. IEEE, 2016, pp. 763-767.

[249] N. Liang and W. Zhang, "Mixed-ADC massive MIMO," IEEE Journal on Selected Areas in Communications, vol. 34, no. 4, pp. 983-997, apr 2016.

[250] J. Choi, D. J. Love, and P. Bidigare, "Downlink training techniques for FDD massive MIMO systems: Open-loop and 
closed-loop training with memory," IEEE Journal of Selected Topics in Signal Processing, vol. 8, no. 5, pp. 802-814, oct 2014.

[251] J. Jose, A. Ashikhmin, T. L. Marzetta, and S. Vishwanath, "Pilot contamination and precoding in multi-cell TDD systems," IEEE Transactions on Wireless Communications, vol. 10, no. 8, pp. 2640-2651, Aug 2011.

[252] M. Alodeh, S. Chatzinotas, and B. Ottersten, "Spatial DCT-based channel estimation in multi-antenna multi-cell interference channels," IEEE Transactions on Signal Processing, vol. 63, no. 6, pp. 1404-1418, Mar 2015.

[253] H. Yin, D. Gesbert, M. Filippou, and Y. Liu, "A coordinated approach to channel estimation in large-scale multiple-antenna systems," IEEE Journal on Selected Areas in Communications, vol. 31, no. 2, pp. 264-273, Feb 2013.

[254] R. Mochaourab, E. Björnson, and M. Bengtsson, "Adaptive pilot clustering in heterogeneous massive MIMO networks," IEEE Transactions on Wireless Communications, vol. 15, no. 8, pp. 5555-5568, Aug 2016.

[255] O. Elijah, C. Y. Leow, T. A. Rahman, S. Nunoo, and S. Z. Iliya, "A comprehensive survey of pilot contamination in massive MIMO- 5G system,” IEEE Communications Surveys \& Tutorials, vol. 18, no. 2, pp. 905-923, 2016.

[256] T. S. Rappaport, S. Sun, R. Mayzus, H. Zhao, Y. Azar, K. Wang, G. N. Wong, J. K. Schulz, M. Samimi, and F. Gutierrez, "Millimeter wave mobile communications for 5G cellular: It will work!” IEEE Access, vol. 1, pp. 335-349, 2013.

[257] S. Rangan, T. S. Rappaport, and E. Erkip, "Millimeter-wave cellular wireless networks: Potentials and challenges," Proceedings of the IEEE, vol. 102, no. 3, pp. 366-385, Mar 2014.

[258] B. Schulz, "LTE transmission modes and beamforming," Rhode and Schwarz white paper, 2011.

[259] ITU-T, Self-FEXT cancellation (vectoring) for use with VDSL2 transceivers, Std., Jan. 2015.

[260] IEEE P802.11ac-2013, IEEE Standard for Information technology-Telecommunications and Information Exchange Between Systems-LAN/MAN-Specific Requirements-Part 11: Wireless LAN Medium Access Control and Physical Layer Specifications-Amendment 4: Enhancements for Very High Throughput for Operation in Bands below 6 GHz, Std.

[261] A. Morello and V. Mignone, "DVB-S2X: the new extensions to the second generation dvb satellite standard DVB-S2," International Journal of Satellite Communications and Networking, vol. 34, no. 3, pp. 323-325, 2016.

[262] A. Checko, H. L. Christiansen, Y. Yan, L. Scolari, G. Kardaras, M. S. Berger, and L. Dittmann, "Cloud RAN for mobile networks: A technology overview," IEEE Communications Surveys \& Tutorials, vol. 17, no. 1, pp. 405-426, 2015. 